Supporting information

\title{
A Study on the Gas-Phase Reactivity of Charged Pyridynes
}

Jacob R. Milton, ${ }^{\#, a}$ Bartłomiej J. Jankiewicz, ${ }^{\#, a, b}$ Joann P. Max, ${ }^{a}$ Nelson R. Vinueza, ${ }^{a}$ Lindsey M.

Kirkpatrick, ${ }^{a, c}$ Karinna Campbell, ${ }^{a}$ Vanessa A. Gallardo, ${ }^{a}$ Jennifer N. Reece, ${ }^{a}$ and Hilkka I.

Kenttämaa ${ }^{a, *}$

\#These authors contributed equally

aPurdue University, Department of Chemistry 560 Oval Drive, West Lafayette, IN 47907-2084, USA

bInstitute of Optoelectronics, Military University of Technology, gen. S. Kaliskiego 2, 00-908 Warsaw, Poland

'Indiana University School of Medicine, Ryan White Center for Pediatric Infectious Disease, 705 Riley Hospital Drive, Room 3032, Indianapolis, Indiana 46202, USA

E-mail: hilkka@purdue.edu; jnash@purdue.edu 


\section{Table of Contents}

Scheme S1. Proposed biradical mechanism for [2+2] cycloaddition of dimethoxyethylene to

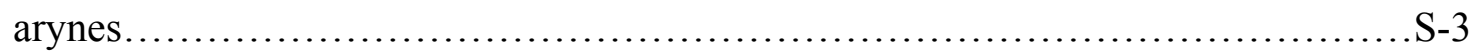

Scheme S2. Reactions of the ortho-benzyne 1 with furan in solution........................

Table S1. Calculated bond angles for the three charged ortho-pyridynes...................... S-3

Figure S1. Potential energy surface calculated at the M062x/aug-cc-pVTZ level of theory for the addition of furan to the 2-pyridyl cation $\mathbf{4}$ and the transition state for acetylene elimination. All values are enthalpies given in $\mathrm{kcal} \mathrm{mol}^{-1}$ and calculated relative to the infinitely separated

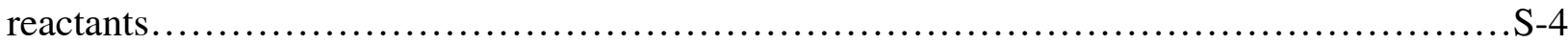

Table S2. Calculated enthalpies of tetrahydrofuran-ortho-pyridyne adducts....................S-4 Scheme S3. Mechanism proposed for ring-cleavage of tetrahydrofuran by the ortho-benzyne 1

Scheme S4. Mechanism proposed for the reaction of perchlorinated ortho-benzyne with diethyl ether.

Figure S2. Reaction kinetics plot for the reaction of the 2,3-didehydropyridinium cation with allyl odide.

Scheme S5. Products formed upon reactions between allylic Grignard reagents and the ortho-

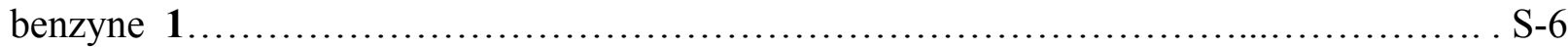

Scheme S5. Reactions proposed for the ortho-benzyne 1 with dimethyldisulfide in liquid ammonia......

Coordinates, calculated energies, zero-point vibrational energies, and thermal corrections to enthalpies, and number of negative frequencies 


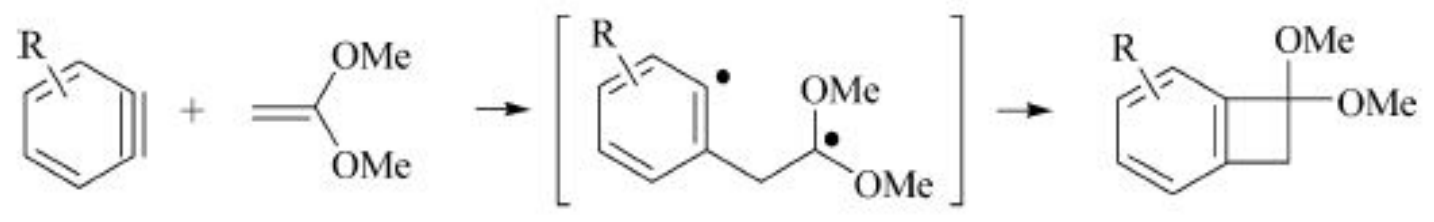

Scheme S1. Proposed mechanism for [2+2] cycloaddition of dimethoxyethylene to arynes, involving the formation of a biradical intermediate. ${ }^{1}$<smiles></smiles><smiles>Oc1cccc2ccccc12</smiles>

Scheme S2. Reactions of ortho-benzyne with furan in solution. ${ }^{2}$

Table S1. Calculated bond angles for the three charged ortho-pyridynes. All values calculated at the B3LYP/aug-cc-pVTZ level of theory, as appropriate for calculating aryne distortion angles. ${ }^{3}$ The with the greatest calculated bond angle for a given charged pyridyne is given in bold. 


\begin{tabular}{|l|l|l|}
\hline Species & Angle & Angle Value (degrees) \\
\hline 2-pyridyl cation & C6-N1-C2 & 117.84578 \\
\hline & N1-C2-C3 & $\mathbf{1 4 1 . 4 1 9 4 7}$ \\
\hline Protonated 2,3-pyridyne & N1-C2-C3 & 121.85815 \\
\hline & C2-C3-C4 & $\mathbf{1 3 1 . 5 5 9 1 1}$ \\
\hline Protonated 3,4-pyridyne & C2-C3-C4 & 120.91219 \\
\hline & C3-C4-C5 & $\mathbf{1 3 2 . 8 3 7 3 9}$ \\
\hline
\end{tabular}

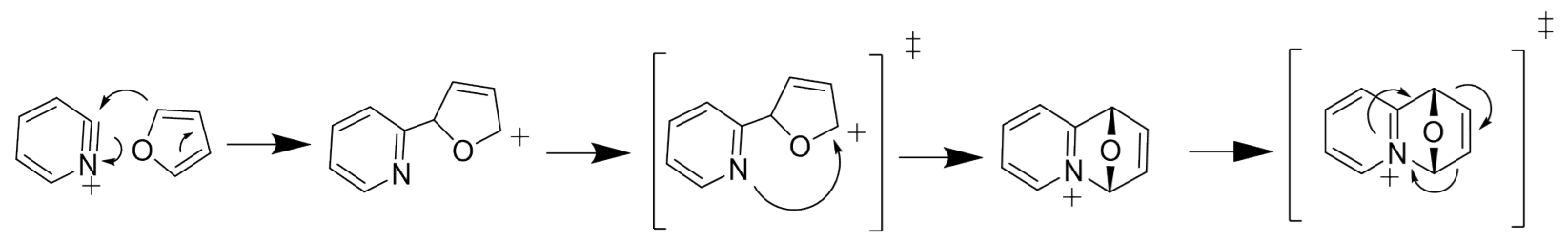

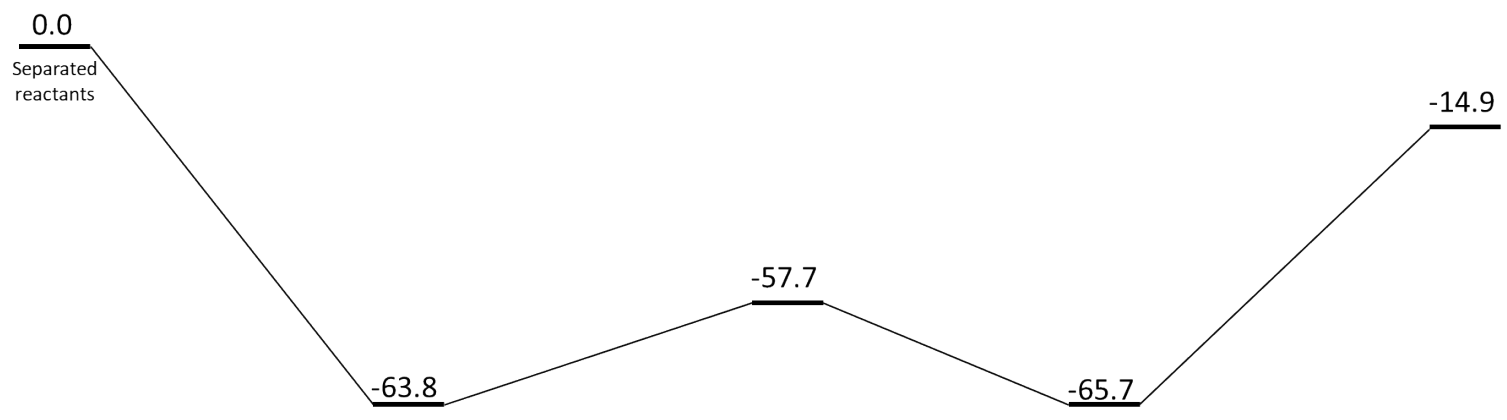

Figure S1. Potential energy surface calculated at the M062x/aug-cc-pVTZ level of theory for the addition of furan to the 2-pyridyl cation $\mathbf{4}$ and the transition state for acetylene elimination. All values are enthalpies given in $\mathrm{kcal} \mathrm{mol}^{-1}$ and calculated relative to the infinitely separated reactants.

Table S2. Calculated enthalpies (in kcal mol-1) for the addition of the 2-pyridyl cation $\mathbf{4}$ and the charged ortho-pyridynes $\mathbf{3}$ and $\mathbf{4}$ to tetrahydrofuran at the M06-2X/aug-cc-pVTZ level of theory. Difference represents the calculated enthalpy of the lower energy species minus that of the higher energy species; the adduct found to be more stable is bolded.

\begin{tabular}{|c|c|l|c|l|c|c|}
\hline Species & 2-pyridyl & Protonated & & Protonated & \\
& cation & & $2,3-$ pyridine & & $3,4-$ \\
4 & & $\mathbf{5}$ & & pyridyne 6 & \\
\hline
\end{tabular}




\begin{tabular}{|c|l|l|l|l|l|l|}
\hline $\begin{array}{c}\text { Addition } \\
\text { site }\end{array}$ & 1 & $\mathbf{2}$ & 2 & $\mathbf{3}$ & $\mathbf{3}$ & $\mathbf{4}$ \\
\hline Enthalpy & $\mathrm{N} / \mathrm{A}^{\mathrm{a}}$ & -300973.9858 & -300919.4303 & -300936.5374 & -300924.564 & -300928.6817 \\
\hline Difference & & N/A & & -17.1 & & -4.1 \\
\hline
\end{tabular}

aNo minimum found.<smiles>CC(O)c1cccc(OCCCCO)c1</smiles>

Scheme S3. Mechanism proposed for ring-cleavage of tetrahydrofuran by the ortho-benzyne in solution. ${ }^{4}$<smiles>C=CCOc1c(Cl)c(Cl)c(Cl)c(Cl)c1Cl</smiles>

Scheme S4. Mechanism proposed for reaction of perchlorinated ortho-benzyne with diethyl ether. ${ }^{5}$ 


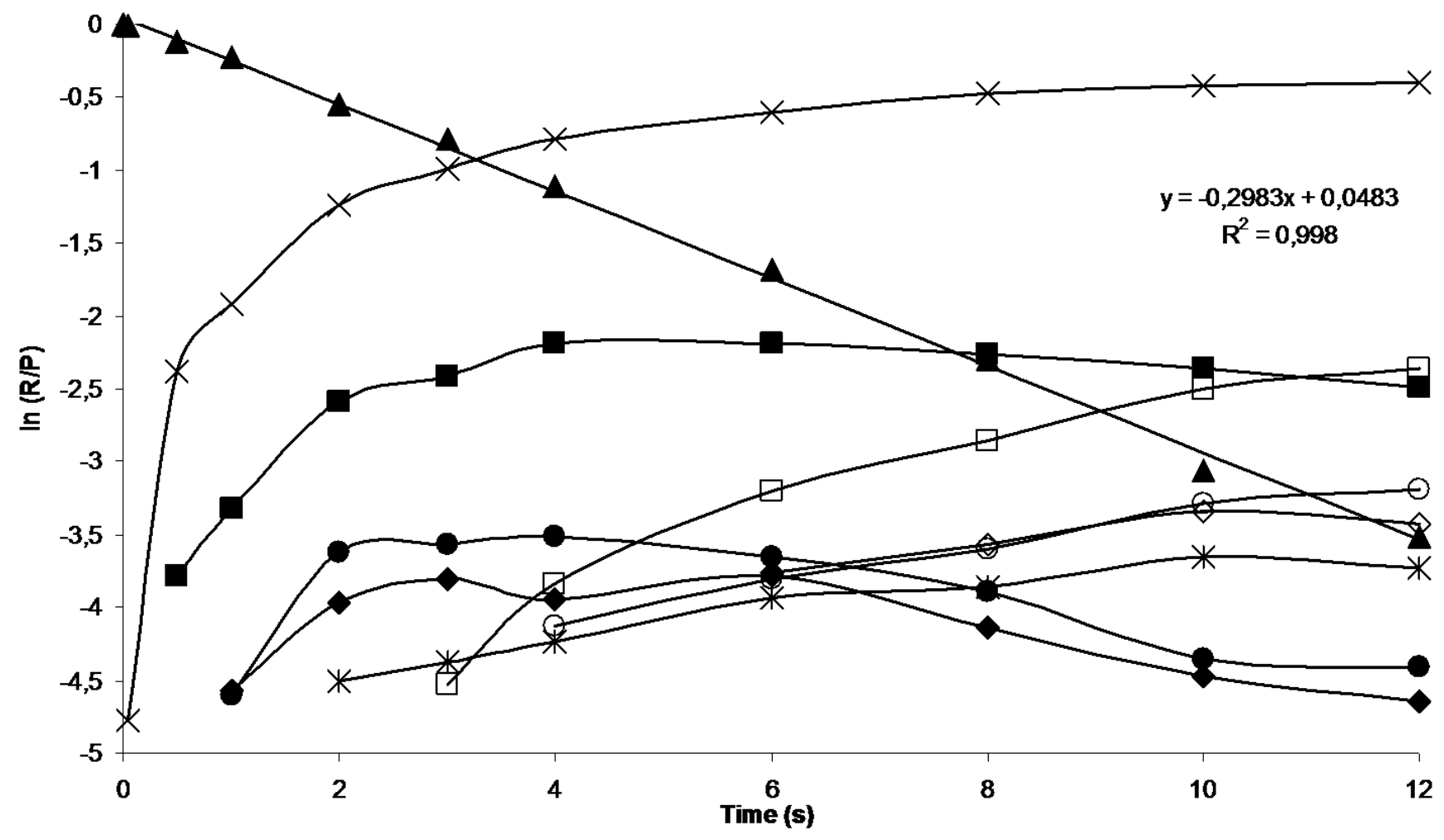

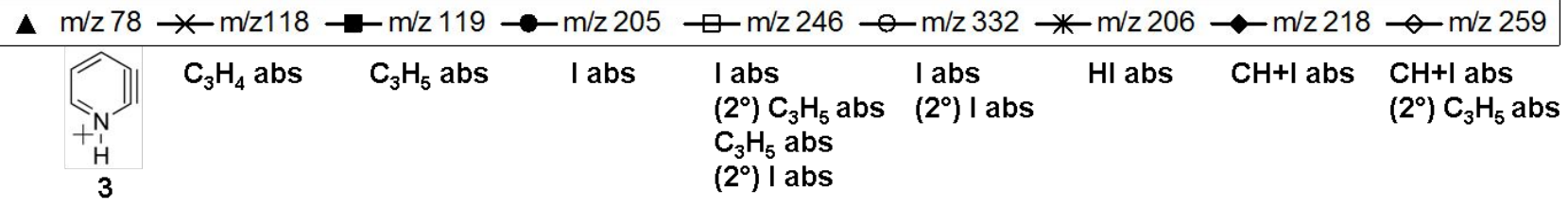

Figure S2. Plot of ln (Reactant ion abundance/Product ion abundance) vs reaction time for reactions of the 2,3-didehydropyridinium cation (5) with allyl iodide. 


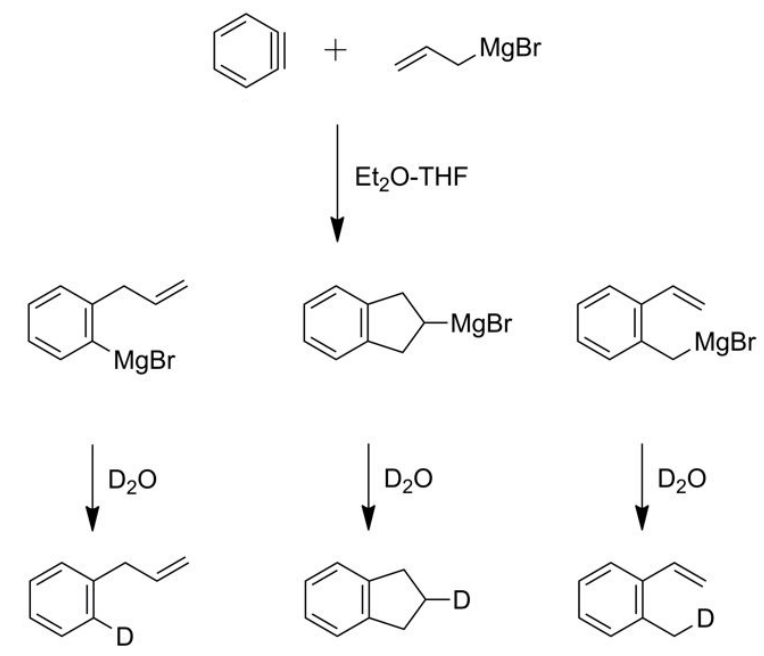

Scheme S5. Products formed upon reactions between allylic Grignard reagents and orthobenzyne. ${ }^{6}$

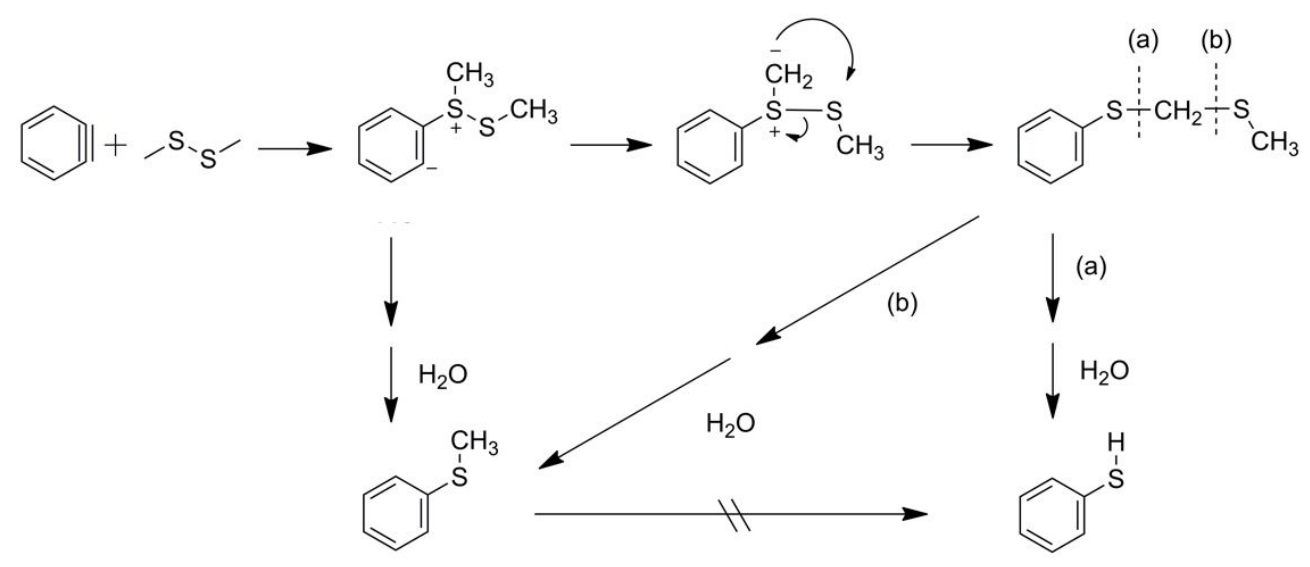

Scheme S6. Reactions proposed for the ortho-benzyne 1 with dimethyldisulfide in liquid ammonia. $^{7}$ 


\section{Computational Data}

All values given in atomic units; coordinates are in Ångstroms. TS = transition state, ZPVE = zero-point vibrational energy.

\begin{tabular}{|l|l|l|l|}
\hline $\begin{array}{l}\text { Addition/elimination of HI } \\
\text { for the 3,4-didehydropyridinium } \\
\text { cation }\end{array}$ & & & \\
\hline Allyl iodide & & & \\
\hline Atomic \# & $\mathrm{X}$ & $\mathrm{Y}$ & $\mathrm{Z}$ \\
\hline 6 & 3.115305 & -0.444562 & -0.255592 \\
\hline 6 & 2.1255 & 0.051264 & 0.472575 \\
\hline 6 & 1.057063 & 0.912466 & -0.085378 \\
\hline 53 & -0.865467 & -0.087891 & 0.009645 \\
\hline 1 & 3.886881 & -1.058967 & 0.186441 \\
\hline 1 & 3.182244 & -0.250983 & -1.319273 \\
\hline 1 & 2.066342 & -0.163668 & 1.533542 \\
\hline 1 & 0.900365 & 1.820423 & 0.486895 \\
\hline 1 & 1.210685 & 1.144372 & -1.133378 \\
\hline & & & \\
\hline Elec energy & -412.912469 & & \\
\hline ZPVE & 0.071044 & & \\
\hline Thermal correction to enthalpy & 0.076958 & & \\
\hline Negative frequencies & 0 & & \\
\hline $3,4-$ didehydropyridinium cation & & & \\
\hline Cs) & & Y & $\mathrm{Z}$ \\
\hline Atomic \# & $\mathrm{X}$ & -0.104387 & 0 \\
\hline 6 & 1.408672 & -1.256644 & 0 \\
\hline 6 & 0.680844 & -1.216098 & 0 \\
\hline 6 & -0.555697 & -0.198374 & 0 \\
\hline 6 & -1.467093 & 0.992253 & 0 \\
\hline 6 & -0.741019 & 1.00123 & 0 \\
\hline 7 & 0.609782 & 0.050764 & 0 \\
\hline 1 & 2.477984 & -0.230672 & 0 \\
\hline 1 & -2.545286 & 1.961345 & 0 \\
\hline 1 & -1.220798 & 1.909452 & 0 \\
\hline 1 & 1.06538 & & \\
\hline & & & \\
\hline & -247.2802836 & & \\
\hline & 0.078225 & & \\
\hline & 0.083466 & & \\
\hline & & & \\
\hline & & & \\
\hline & & & \\
\hline & & & \\
\hline & & & \\
\hline & & & \\
\hline & & & \\
\hline & & & \\
\hline & & & \\
\hline & & & \\
\hline & & & \\
\hline & & & \\
\hline & & & \\
\hline
\end{tabular}




\begin{tabular}{|c|c|c|c|}
\hline & 0 & & \\
\hline \multicolumn{4}{|c|}{ Intermediate } \\
\hline Atomic \# & $\mathrm{X}$ & $\mathrm{Y}$ & $Z$ \\
\hline 6 & -2.823131 & -1.399196 & 0.168685 \\
\hline 6 & -3.413034 & 1.349481 & -0.207282 \\
\hline 6 & -4.366405 & 0.358328 & -0.343835 \\
\hline 7 & -4.054487 & -0.93841 & -0.160955 \\
\hline 1 & -2.716892 & -2.466958 & 0.291576 \\
\hline 1 & -3.680624 & 2.384819 & -0.356902 \\
\hline 1 & -5.395528 & 0.561021 & -0.601108 \\
\hline 1 & -4.798165 & -1.616676 & -0.276165 \\
\hline 6 & -1.855898 & -0.446515 & 0.310926 \\
\hline 6 & -2.14564 & 0.902916 & 0.118159 \\
\hline 6 & -0.729369 & 1.337872 & 0.39501 \\
\hline 1 & -0.603405 & 1.994307 & 1.25346 \\
\hline 1 & -0.217762 & 1.772144 & -0.464065 \\
\hline 6 & -0.391804 & -0.17867 & 0.635179 \\
\hline 6 & 0.620577 & -0.794237 & -0.303925 \\
\hline 1 & -0.144724 & -0.396975 & 1.672805 \\
\hline 1 & 0.714266 & -1.865416 & -0.156301 \\
\hline 1 & 0.385858 & -0.586048 & -1.344615 \\
\hline \multirow[t]{5}{*}{53} & 2.558445 & 0.040486 & 0.059597 \\
\hline & -660.345576 & & \\
\hline & 0.155934 & & \\
\hline & 0.165548 & & \\
\hline & 0 & & \\
\hline \multicolumn{4}{|c|}{ Iodine proton trans TS } \\
\hline Atomic \# & $\mathrm{X}$ & $\mathrm{Y}$ & $\mathrm{Z}$ \\
\hline 6 & 2.175737 & -1.275628 & 0.704591 \\
\hline 6 & 3.615338 & 0.674474 & -0.784987 \\
\hline 6 & 4.112796 & -0.597845 & -0.534725 \\
\hline 7 & 3.403176 & -1.492574 & 0.171154 \\
\hline 1 & 1.71852 & -2.091874 & 1.24226 \\
\hline 1 & 4.194907 & 1.380756 & -1.360061 \\
\hline 1 & 5.075423 & -0.934361 & -0.889287 \\
\hline 1 & 3.818991 & -2.405823 & 0.312311 \\
\hline 6 & 1.657693 & -0.026789 & 0.482813 \\
\hline 6 & 2.371189 & 0.927293 & -0.259241 \\
\hline
\end{tabular}




\begin{tabular}{|c|c|c|c|}
\hline 6 & 1.2735 & 1.966686 & -0.142576 \\
\hline 1 & 0.827637 & 2.270776 & -1.089089 \\
\hline 1 & 1.551659 & 2.84712 & 0.435014 \\
\hline 6 & 0.51401 & 0.874786 & 0.645148 \\
\hline 6 & -0.620636 & 0.881638 & 1.464135 \\
\hline 1 & -0.510418 & 0.398996 & -0.054121 \\
\hline 1 & -0.816844 & 0.079784 & 2.160043 \\
\hline 1 & -1.218833 & 1.777668 & 1.547838 \\
\hline \multirow[t]{5}{*}{53} & -2.441037 & -0.239123 & -0.263283 \\
\hline & -660.2429329 & & \\
\hline & 0.147064 & & \\
\hline & 0.156206 & & \\
\hline & 1 & & \\
\hline \multicolumn{4}{|c|}{ HI (CinfV) } \\
\hline Atomic \# & $\mathrm{X}$ & $\mathrm{Y}$ & $\mathrm{Z}$ \\
\hline 53 & -1.737662 & 0.358025 & 0 \\
\hline \multirow[t]{5}{*}{1} & -3.349128 & 0.358025 & 0 \\
\hline & -296.217984 & & \\
\hline & 0.00536 & & \\
\hline & 0.008665 & & \\
\hline & 1 & & \\
\hline \multicolumn{4}{|c|}{ Final ionic product } \\
\hline Atomic \# & $\mathrm{X}$ & $\mathrm{Y}$ & $Z$ \\
\hline 6 & -0.632217 & -1.431577 & 0 \\
\hline 6 & -1.38179 & 1.308755 & 0 \\
\hline 6 & -2.308467 & 0.280531 & 0 \\
\hline 7 & -1.918501 & -1.007548 & 0 \\
\hline 1 & -0.465569 & -2.498024 & 0 \\
\hline 1 & -1.711828 & 2.336686 & 0 \\
\hline 1 & -3.375189 & 0.447786 & 0 \\
\hline 1 & -2.646302 & -1.712588 & 0 \\
\hline 6 & 0.313501 & -0.442341 & 0 \\
\hline 6 & -0.06271 & 0.907619 & 0 \\
\hline 6 & 1.373202 & 1.391429 & 0 \\
\hline 1 & 1.663216 & 1.947739 & 0.890418 \\
\hline 1 & 1.663216 & 1.947739 & -0.890418 \\
\hline 6 & 1.750154 & -0.103548 & 0 \\
\hline 6 & 2.878909 & -0.788159 & 0 \\
\hline
\end{tabular}




\begin{tabular}{|c|c|c|c|}
\hline 1 & 3.835086 & -0.283152 & 0 \\
\hline 1 & 2.883473 & -1.869585 & 0 \\
\hline & -364.089162 & & \\
\hline & 0.142232 & & \\
\hline & 0.150066 & & \\
\hline & 0 & & \\
\hline \multicolumn{4}{|l|}{$\begin{array}{l}\text { Iodide abstraction reaction } \\
\text { for 2-pyridyl cation }\end{array}$} \\
\hline \multicolumn{4}{|l|}{ Intermediate } \\
\hline Atomic \# & $\mathrm{X}$ & $\mathrm{Y}$ & $\mathrm{Z}$ \\
\hline 6 & -3.300934 & -1.159522 & -0.495015 \\
\hline 6 & -2.525363 & -0.176905 & -1.075198 \\
\hline 6 & -2.726737 & 1.097282 & -0.560953 \\
\hline 6 & -3.648336 & 1.28948 & 0.459552 \\
\hline 6 & -4.351389 & 0.200264 & 0.945472 \\
\hline 7 & -4.16816 & -1.032453 & 0.451617 \\
\hline 1 & -2.161199 & 1.929103 & -0.957776 \\
\hline 1 & -1.81496 & -0.377052 & -1.863182 \\
\hline 1 & -3.819769 & 2.271341 & 0.87522 \\
\hline 1 & -5.077894 & 0.296271 & 1.740088 \\
\hline 53 & -3.082196 & -3.184964 & -1.247585 \\
\hline 6 & -4.216737 & -4.166199 & 0.422058 \\
\hline 1 & -4.605713 & -3.249299 & 0.862958 \\
\hline 1 & -3.420818 & -4.616058 & 1.005041 \\
\hline 6 & -5.201166 & -5.104416 & -0.127262 \\
\hline 6 & -5.059437 & -6.418109 & 0.012913 \\
\hline 1 & -4.189061 & -6.846472 & 0.495322 \\
\hline 1 & -6.074558 & -4.683174 & -0.608871 \\
\hline 1 & -5.820419 & -7.103626 & -0.332643 \\
\hline Elec energy & -660.287228 & & \\
\hline ZPVE & \begin{tabular}{|l|l|}
0.150041 \\
\end{tabular} & & \\
\hline Thermal correction to enthalpy & 0.160529 & & \\
\hline Negative frequencies & 1 & & \\
\hline \multicolumn{4}{|l|}{ 2-iodopyridine (Cs) } \\
\hline Atomic \# & $\mathrm{X}$ & $\mathrm{Y}$ & $\mathrm{Z}$ \\
\hline 6 & 0.579453 & 0.079767 & 0 \\
\hline 6 & 1.362171 & 1.229587 & 0 \\
\hline
\end{tabular}




\begin{tabular}{|c|c|c|c|}
\hline 6 & \begin{tabular}{|l|}
2.735285 \\
\end{tabular} & 1.061226 & 0 \\
\hline 6 & 3.260404 & -0.223544 & 0 \\
\hline 6 & 2.380048 & -1.291344 & 0 \\
\hline 7 & \begin{tabular}{|l|}
1.053197 \\
\end{tabular} & -1.143925 & 0 \\
\hline 1 & 3.386704 & 1.924685 & 0 \\
\hline 1 & 0.908001 & 2.208399 & 0 \\
\hline 1 & 4.326861 & -0.394886 & 0 \\
\hline 1 & 2.741675 & -2.311912 & 0 \\
\hline \multirow[t]{5}{*}{53} & -1.521155 & 0.287287 & 0 \\
\hline & -543.290559 & & \\
\hline & 0.078713 & & \\
\hline & 0.085366 & & \\
\hline & 0 & & \\
\hline \multicolumn{4}{|c|}{ 2-pyridyl cation (Cs) } \\
\hline Atomic \# & $X$ & $\mathrm{Y}$ & $\mathrm{Z}$ \\
\hline 6 & -0.095472 & -1.179166 & 0 \\
\hline 6 & -1.345404 & -0.673643 & 0 \\
\hline 6 & -1.08922 & 0.715739 & 0 \\
\hline 6 & \begin{tabular}{|l|l|}
0.196519 \\
\end{tabular} & 1.276286 & 0 \\
\hline 6 & \begin{tabular}{|l|}
1.324814 \\
\end{tabular} & 0.475631 & 0 \\
\hline 7 & 1.047965 & -0.864682 & 0 \\
\hline 1 & -1.960507 & 1.361414 & 0 \\
\hline 1 & -2.309082 & -1.15426 & 0 \\
\hline 1 & 0.313491 & 2.350967 & 0 \\
\hline \multirow[t]{5}{*}{1} & 2.35386 & 0.798234 & 0 \\
\hline & -247.301944 & & \\
\hline & 0.076069 & & \\
\hline & 0.081361 & & \\
\hline & 0 & & \\
\hline \multicolumn{4}{|c|}{ Allyl cation $(\mathrm{C} 2 \mathrm{~V})$} \\
\hline Atomic \# & $\mathrm{X}$ & $\mathrm{Y}$ & $\mathrm{Z}$ \\
\hline 6 & 0 & -1.174047 & -0.198209 \\
\hline 1 & 0 & -1.164325 & -1.283335 \\
\hline 1 & 0 & -2.141535 & 0.292476 \\
\hline 6 & 0 & 0 & 0.516328 \\
\hline 6 & 0 & 1.174047 & -0.198209 \\
\hline 1 & 0 & 1.164325 & -1.283335 \\
\hline
\end{tabular}




\begin{tabular}{|c|c|c|c|}
\hline 1 & 0 & 0 & 1.597585 \\
\hline 1 & 0 & 2.141535 & 0.292476 \\
\hline & -116.940161 & & \\
\hline & 0.068625 & & \\
\hline & 0.073407 & & \\
\hline & 0 & & \\
\hline \multicolumn{4}{|l|}{$\begin{array}{l}\text { Addition/thioformaldehyde } \\
\text { elimination reaction of } 3,4- \\
\text { didehydropyridinium cation }\end{array}$} \\
\hline \multicolumn{4}{|l|}{$\begin{array}{l}\text { 3,4-didehydropyridinium cation } \\
\text { (Cs) }\end{array}$} \\
\hline Atomic \# & $X$ & $\mathrm{Y}$ & $\mathrm{Z}$ \\
\hline 6 & 1.408672 & -0.104387 & 0 \\
\hline 6 & 0.680844 & -1.256644 & 0 \\
\hline 6 & -0.555697 & -1.216098 & 0 \\
\hline 6 & -1.467093 & -0.198374 & 0 \\
\hline 6 & -0.741019 & 0.992253 & 0 \\
\hline 7 & 0.609782 & 1.00123 & 0 \\
\hline 1 & \begin{tabular}{|l|}
2.477984 \\
\end{tabular} & 0.050764 & 0 \\
\hline 1 & -2.545286 & -0.230672 & 0 \\
\hline 1 & -1.220798 & 1.961345 & 0 \\
\hline 1 & 1.06538 & 1.909452 & 0 \\
\hline Elec energy & -247.2802836 & & \\
\hline ZPVE & 0.078225 & & \\
\hline Thermal correction to enthalpy & 0.083466 & & \\
\hline Negative frequencies & 0 & & \\
\hline \multicolumn{4}{|l|}{ Dimethyl disulfide (C2) } \\
\hline Atomic \# & $\mathrm{X}$ & $\mathrm{Y}$ & $\mathrm{Z}$ \\
\hline 16 & -0.545541 & 0.865888 & -0.991211 \\
\hline 16 & 0.545541 & -0.865888 & -0.991211 \\
\hline 6 & -0.265617 & -1.824444 & 0.318427 \\
\hline 1 & -1.31291 & -1.977924 & 0.075342 \\
\hline 1 & 0.247139 & -2.784286 & 0.36021 \\
\hline 1 & -0.168008 & -1.32382 & 1.278113 \\
\hline 6 & 0.265617 & 1.824444 & 0.318427 \\
\hline 1 & 1.31291 & 1.977924 & 0.075342 \\
\hline 1 & -0.247139 & 2.784286 & 0.36021 \\
\hline 1 & 0.168008 & 1.32382 & 1.278113 \\
\hline
\end{tabular}




\begin{tabular}{|c|c|c|c|}
\hline \multicolumn{4}{|c|}{ 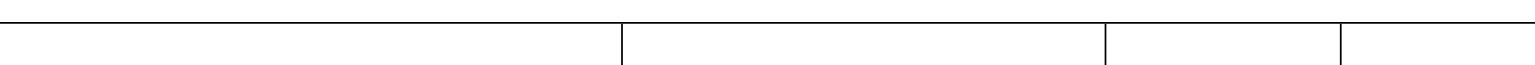 } \\
\hline & & & \\
\hline & 0.077856 & & \\
\hline & 0.085227 & & \\
\hline & 0 & & \\
\hline \multicolumn{4}{|c|}{ Intermediate } \\
\hline Atomic \# & $\mathrm{X}$ & $\mathrm{Y}$ & $\mathrm{Z}$ \\
\hline 6 & -0.919254 & 1.094182 & -0.187659 \\
\hline 6 & -1.559939 & -0.134368 & -0.064282 \\
\hline 6 & -0.641278 & -1.156187 & 0.025541 \\
\hline 6 & 0.74922 & -1.079544 & 0.015029 \\
\hline 6 & 1.273071 & 0.185439 & -0.104811 \\
\hline 7 & 0.426837 & 1.223451 & -0.201262 \\
\hline 1 & -1.445804 & 2.036947 & -0.275414 \\
\hline 1 & 1.420218 & -1.922717 & 0.091794 \\
\hline 1 & 2.329511 & 0.402209 & -0.127288 \\
\hline 1 & 0.824102 & 2.152578 & -0.286497 \\
\hline 16 & -1.475325 & -2.772307 & 0.239335 \\
\hline 16 & -2.628446 & -2.954358 & -1.464058 \\
\hline 6 & -4.203324 & -2.329954 & -0.813081 \\
\hline 1 & -4.079679 & -1.29195 & -0.522141 \\
\hline 1 & -4.884572 & -2.414247 & -1.658899 \\
\hline 1 & -4.552249 & -2.955379 & 0.002424 \\
\hline 6 & -0.226153 & -4.033477 & -0.083846 \\
\hline 1 & 0.252658 & -3.847119 & -1.041856 \\
\hline 1 & 0.488192 & -3.999168 & 0.735347 \\
\hline \multirow[t]{5}{*}{1} & -0.743581 & -4.988605 & -0.085779 \\
\hline & -1123.536424 & & \\
\hline & 0.15889 & & \\
\hline & 0.171043 & & \\
\hline & 0 & & \\
\hline \multicolumn{4}{|c|}{ Transition state } \\
\hline Atomic \# & $\mathrm{X}$ & $\mathrm{Y}$ & $\mathrm{Z}$ \\
\hline 6 & 2.158429 & -1.304821 & -0.36692 \\
\hline 6 & 0.872719 & -0.907965 & -0.668764 \\
\hline 6 & 0.556927 & 0.389508 & -0.275204 \\
\hline 6 & 1.417294 & 1.238304 & 0.428798 \\
\hline 6 & 2.71095 & 0.809342 & 0.563729 \\
\hline 7 & 3.050763 & -0.426357 & 0.139775 \\
\hline 1 & 2.537312 & -2.306256 & -0.524757 \\
\hline
\end{tabular}




\begin{tabular}{|c|c|c|c|}
\hline 1 & 1.131062 & 2.208319 & 0.808356 \\
\hline 1 & 3.498127 & 1.408919 & \begin{tabular}{|l|}
0.993986 \\
\end{tabular} \\
\hline 1 & 4.019597 & -0.709865 & 0.228064 \\
\hline 16 & -1.075306 & 0.767484 & -0.777597 \\
\hline 16 & -2.220003 & -0.72751 & \begin{tabular}{|l|}
0.540781 \\
\end{tabular} \\
\hline 6 & -1.363584 & -2.184066 & 0.227006 \\
\hline 1 & -0.338666 & -1.796931 & -0.40334 \\
\hline 1 & -0.951057 & -2.621519 & 1.129862 \\
\hline 1 & -1.876612 & -2.879485 & -0.428556 \\
\hline 6 & -1.565793 & 2.214457 & 0.175421 \\
\hline 1 & -1.309354 & 2.071769 & 1.221637 \\
\hline 1 & -1.082198 & 3.089295 & -0.251628 \\
\hline \multirow[t]{5}{*}{1} & -2.644422 & 2.299284 & 0.061657 \\
\hline & -1123.51426 & & \\
\hline & 0.154452 & & \\
\hline & 0.165579 & & \\
\hline & 0 & & \\
\hline \multicolumn{4}{|l|}{$\begin{array}{l}\text { 4-Methylsulfanylpyridinium } \\
\text { cation }\end{array}$} \\
\hline Atomic \# & $\mathrm{X}$ & $\mathrm{Y}$ & $\mathrm{Z}$ \\
\hline 6 & -1.179509 & 0.607716 & -0.010803 \\
\hline 6 & -1.265246 & -0.750347 & 0.035883 \\
\hline 6 & -0.089713 & -1.532299 & 0.014287 \\
\hline 6 & 1.146021 & -0.863773 & -0.055928 \\
\hline 6 & 1.173229 & 0.502251 & -0.100602 \\
\hline 7 & 0.028868 & 1.208081 & -0.077782 \\
\hline 1 & -2.042566 & 1.255923 & 0.002688 \\
\hline 1 & 2.081414 & -1.399368 & -0.075822 \\
\hline 1 & 2.090579 & 1.069302 & -0.154976 \\
\hline 1 & 0.073742 & 2.217903 & -0.111674 \\
\hline 16 & -0.284566 & -3.236102 & \begin{tabular}{|l}
0.076605 \\
\end{tabular} \\
\hline 6 & 1.406016 & -3.863528 & \begin{tabular}{|l|}
0.029565 \\
\end{tabular} \\
\hline 1 & 1.896232 & -3.584558 & -0.899528 \\
\hline 1 & 1.967514 & -3.526958 & \begin{tabular}{|l|}
0.897183 \\
\end{tabular} \\
\hline 1 & 1.304507 & -4.944954 & \begin{tabular}{|l|}
0.068183 \\
\end{tabular} \\
\hline \multirow[t]{3}{*}{1} & -2.237378 & -1.218872 & 0.08929 \\
\hline & -686.144415 & & \\
\hline & 0.132494 & & \\
\hline
\end{tabular}




\begin{tabular}{|c|c|c|c|}
\hline & 0.140832 & & \\
\hline & 0 & & \\
\hline \multicolumn{4}{|c|}{ Thioformaldehyde (C2V) } \\
\hline Atomic \# & $\mathrm{X}$ & $\mathrm{Y}$ & $\mathrm{Z}$ \\
\hline 6 & 0 & 0 & -1.019451 \\
\hline 1 & 0 & 0.921611 & -1.595323 \\
\hline 1 & 0 & -0.921611 & -1.595323 \\
\hline \multirow[t]{5}{*}{16} & 0 & 0 & 0.58171 \\
\hline & \begin{tabular}{|l|}
-437.455268 \\
\end{tabular} & & \\
\hline & 0.024959 & & \\
\hline & 0.028831 & & \\
\hline & 0 & & \\
\hline \multicolumn{4}{|c|}{$\begin{array}{l}\text { Additon/thiomethyl radical } \\
\text { elimination reaction of } 3,4- \\
\text { didehydropyridinium cation }\end{array}$} \\
\hline \multicolumn{4}{|c|}{ res } \\
\hline Atomic \# & $\mathrm{X}$ & $\mathrm{Y}$ & $\mathrm{Z}$ \\
\hline 6 & 2.812826 & 0.841849 & 0.455059 \\
\hline 6 & 1.485774 & 1.106644 & 0.202329 \\
\hline 6 & 0.754865 & 0.050868 & -0.249254 \\
\hline 6 & 1.23094 & -1.231279 & -0.479594 \\
\hline 6 & 2.573025 & -1.418147 & -0.245129 \\
\hline 7 & 3.313153 & -0.388625 & 0.206908 \\
\hline 1 & 3.524993 & 1.556177 & 0.846547 \\
\hline 1 & 0.615 & -2.051587 & -0.820888 \\
\hline 1 & \begin{tabular}{|l|}
3.086289 \\
\end{tabular} & -2.355045 & -0.398938 \\
\hline 1 & 4.300473 & -0.550834 & 0.370939 \\
\hline 16 & -1.009752 & 0.57177 & -0.639572 \\
\hline 16 & -1.911966 & -0.799793 & 0.645135 \\
\hline 6 & -3.595971 & -0.706355 & -0.018681 \\
\hline 1 & -3.617596 & -1.015288 & -1.059054 \\
\hline 1 & -4.167904 & -1.409462 & 0.584241 \\
\hline 1 & -4.000455 & 0.292786 & 0.112525 \\
\hline 6 & -0.926499 & 2.149063 & 0.038415 \\
\hline 1 & 0.475137 & 2.0805 & 0.20646 \\
\hline 1 & -1.356052 & 2.867529 & -0.648698 \\
\hline 1 & -1.314222 & 2.198103 & 1.05064 \\
\hline Elec energy & \begin{tabular}{|l|}
-1123.521064 \\
\end{tabular} & & \\
\hline ZPVE & 0.154272 & & \\
\hline
\end{tabular}




\begin{tabular}{|c|c|c|c|}
\hline Thermal correction to enthalpy & 0.165809 & & \\
\hline Negative frequencies & 1 & & \\
\hline \multicolumn{4}{|l|}{ 4-Thiomethylpyridinium cation } \\
\hline Atomic \# & $\mathrm{X}$ & $\mathrm{Y}$ & $\mathrm{Z}$ \\
\hline 6 & 2.04362 & -1.007288 & -0.051961 \\
\hline 6 & 0.706334 & -1.263748 & -0.025321 \\
\hline 6 & -0.210815 & -0.193607 & 0.03003 \\
\hline 6 & 0.293859 & 1.119146 & 0.069368 \\
\hline 6 & 1.645164 & 1.317553 & 0.03934 \\
\hline 7 & 2.48819 & 0.269753 & -0.024849 \\
\hline 1 & 2.793971 & -1.781682 & -0.098511 \\
\hline 1 & -0.353901 & 1.976996 & 0.14812 \\
\hline 1 & 2.094433 & 2.298896 & 0.07183 \\
\hline 1 & 3.484386 & 0.442532 & -0.048942 \\
\hline 16 & -1.879037 & -0.613897 & 0.055541 \\
\hline 6 & -2.745326 & 0.830151 & -0.105013 \\
\hline 1 & -2.322932 & 1.725646 & -0.527572 \\
\hline 1 & -3.807541 & 0.746019 & 0.056058 \\
\hline \multirow[t]{5}{*}{1} & 0.361834 & -2.287563 & -0.054356 \\
\hline & -685.478246 & & \\
\hline & 0.118027 & & \\
\hline & 0.126549 & & \\
\hline & 0 & & \\
\hline \multicolumn{4}{|l|}{ Thiomethyl radical } \\
\hline Atomic \# & $\mathrm{X}$ & $\mathrm{Y}$ & $\mathrm{Z}$ \\
\hline 16 & -2.019758 & -0.235156 & -0.02881 \\
\hline 6 & -0.221817 & -0.18467 & -0.028993 \\
\hline 1 & 0.119646 & -1.224634 & -0.028821 \\
\hline 1 & 0.164016 & 0.297233 & -0.926163 \\
\hline \multirow[t]{5}{*}{1} & 0.163871 & 0.297121 & 0.868335 \\
\hline & -437.999371 & & \\
\hline & 0.035654 & & \\
\hline & 0.03985 & & \\
\hline & 0 & & \\
\hline $\begin{array}{l}\text { Addition/methanethiol } \\
\text { elimination reaction of 2,3- } \\
\text { didehydropyridinium cation }\end{array}$ & & & \\
\hline
\end{tabular}




\begin{tabular}{|c|c|c|c|}
\hline $\begin{array}{l}\text { 2,3-didehydropyridinium cation } \\
\text { (Cs) }\end{array}$ & & & \\
\hline Atomic \# & $\mathrm{X}$ & $\mathrm{Y}$ & Z \\
\hline 6 & -0.6257 & 1.063063 & 0 \\
\hline 6 & 0.757391 & 1.026284 & 0 \\
\hline 6 & 1.482845 & -0.188869 & 0 \\
\hline 6 & 0.57989 & -1.211902 & 0 \\
\hline 6 & -0.65388 & -1.201186 & 0 \\
\hline 7 & -1.362588 & -0.08471 & 0 \\
\hline 1 & 2.562423 & -0.234911 & 0 \\
\hline 1 & -1.185277 & 1.987732 & 0 \\
\hline 1 & 1.296227 & 1.964309 & 0 \\
\hline 1 & -2.378533 & -0.0485 & 0 \\
\hline Elec energy & -247.262585 & & \\
\hline ZPVE & 0.077725 & & \\
\hline Thermal correction to enthalpy & 0.083021 & & \\
\hline Negative frequencies & 0 & & \\
\hline Hydride abstraction TS & & & \\
\hline Atomic \# & $\mathrm{X}$ & $\mathrm{Y}$ & $Z$ \\
\hline 6 & -3.531622 & -0.515777 & -0.353165 \\
\hline 6 & -2.533653 & -0.26373 & -1.272494 \\
\hline 6 & -1.315359 & 0.337242 & -0.887675 \\
\hline 6 & -1.357008 & 0.585149 & 0.448556 \\
\hline 6 & -2.2283 & 0.376149 & 1.332734 \\
\hline 7 & -3.374226 & -0.191888 & 0.956346 \\
\hline 1 & -0.506402 & 0.550706 & -1.575267 \\
\hline 1 & -4.470515 & -0.976603 & -0.624541 \\
\hline 1 & -2.693572 & -0.53701 & -2.305844 \\
\hline 1 & -4.122399 & -0.390313 & 1.613325 \\
\hline 16 & 2.049718 & 1.042631 & -0.574158 \\
\hline 16 & 1.855188 & -0.993555 & -0.415479 \\
\hline 6 & 3.24523 & -1.439751 & 0.662013 \\
\hline 1 & 3.140576 & -0.979719 & 1.640216 \\
\hline 1 & 3.202357 & -2.522618 & 0.764035 \\
\hline 1 & 4.180482 & -1.150835 & \begin{tabular}{|l|}
0.193446 \\
\end{tabular} \\
\hline 6 & 1.235394 & 1.602149 & \begin{tabular}{|l|}
0.913639 \\
\end{tabular} \\
\hline 1 & 1.347409 & 2.681911 & \begin{tabular}{|l|}
0.997364 \\
\end{tabular} \\
\hline 1 & 0.130746 & 1.38662 & \begin{tabular}{|l|}
0.853079 \\
\end{tabular} \\
\hline 1 & 1.59711 & 1.098912 & 1.806546 \\
\hline
\end{tabular}




\begin{tabular}{|c|c|c|c|}
\hline & & & \\
\hline & -1123.475403 & & \\
\hline & 0.155041 & & \\
\hline & 0.167522 & & \\
\hline & 0 & & \\
\hline Addition II & & & \\
\hline Atomic \# & $\mathrm{X}$ & $\mathrm{Y}$ & $\mathrm{Z}$ \\
\hline 6 & -1.07974 & -1.432071 & -0.552726 \\
\hline 6 & -2.026688 & -0.452778 & -0.712067 \\
\hline 6 & -1.763867 & 0.814073 & -0.196763 \\
\hline 6 & -0.573316 & 1.067778 & 0.46319 \\
\hline 6 & 0.356929 & 0.053377 & 0.613431 \\
\hline 7 & 0.06179 & -1.147271 & 0.095924 \\
\hline 1 & -2.491628 & 1.605201 & -0.315397 \\
\hline 1 & -1.184194 & -2.440148 & -0.924685 \\
\hline 1 & -2.94514 & -0.673398 & -1.233641 \\
\hline 1 & 0.784064 & -1.863609 & 0.199885 \\
\hline 16 & 2.916281 & -1.033491 & 0.989552 \\
\hline 6 & 1.656231 & 0.226191 & 1.350696 \\
\hline 1 & -0.354282 & 2.045972 & 0.866173 \\
\hline 1 & 1.465289 & 0.157312 & 2.425102 \\
\hline 16 & 3.367074 & -0.626547 & -0.973125 \\
\hline 1 & 2.047705 & 1.222 & 1.152037 \\
\hline 6 & \begin{tabular}{|l|}
4.66959 \\
\end{tabular} & 0.625587 & -0.810615 \\
\hline 1 & 5.505481 & 0.218614 & -0.250732 \\
\hline 1 & \begin{tabular}{|l|}
4.98347 \\
\end{tabular} & 0.850396 & -1.828575 \\
\hline 1 & 4.288084 & 1.528325 & -0.342694 \\
\hline & -1123.655907 & & \\
\hline & 0.161955 & & \\
\hline & 0.173424 & & \\
\hline & 0 & & \\
\hline Proton Tra & & & \\
\hline Atomic \# & $\mathrm{X}$ & $\mathrm{Y}$ & $\mathrm{Z}$ \\
\hline 6 & -2.692666 & -1.253819 & -0.304255 \\
\hline 6 & -3.648079 & -0.307524 & -0.477775 \\
\hline 6 & -3.294666 & 1.039932 & -0.247068 \\
\hline 6 & -2.027183 & 1.377726 & 0.1289 \\
\hline 6 & -1.035756 & 0.380946 & 0.291349 \\
\hline 7 & -1.435656 & $\begin{array}{l}-0.895778 \\
\end{array}$ & 0.069374 \\
\hline 1 & -4.040568 & 1.814028 & -0.364414 \\
\hline
\end{tabular}




\begin{tabular}{|c|c|c|c|}
\hline 1 & -2.859698 & -2.311598 & -0.438373 \\
\hline 1 & -4.645768 & -0.590177 & -0.772413 \\
\hline 1 & -0.748554 & -1.62506 & 0.220092 \\
\hline 16 & 1.438242 & -0.576251 & 1.061038 \\
\hline 6 & 0.310644 & 0.693778 & 0.608643 \\
\hline 1 & -1.751026 & 2.405867 & 0.31009 \\
\hline 1 & 0.487252 & 1.668621 & 1.041287 \\
\hline 16 & 2.45542 & -0.392688 & -0.804679 \\
\hline 1 & 1.2779 & 0.54072 & -0.792302 \\
\hline 6 & 3.756404 & 0.805851 & -0.429423 \\
\hline 1 & 4.512975 & 0.29673 & 0.160598 \\
\hline 1 & 4.177697 & 1.121125 & -1.381913 \\
\hline \multirow[t]{5}{*}{1} & 3.331071 & 1.645085 & 0.114051 \\
\hline & -1123.577249 & & \\
\hline & 0.155601 & & \\
\hline & 0.16679 & & \\
\hline & 0 & & \\
\hline \multicolumn{4}{|c|}{ Methanethiol (Cs) } \\
\hline Atomic \# & $\mathrm{X}$ & $\mathrm{Y}$ & $\mathrm{Z}$ \\
\hline 16 & 0.116139 & 0.667979 & 0 \\
\hline 6 & -0.023802 & -1.142429 & 0 \\
\hline 1 & -0.531886 & -1.496352 & -0.891849 \\
\hline 1 & 0.993665 & -1.524165 & 0 \\
\hline 1 & -0.531886 & -1.496352 & 0.891849 \\
\hline \multirow[t]{5}{*}{1} & -1.19618 & 0.932775 & 0 \\
\hline & -438.690193 & & \\
\hline & 0.046093 & & \\
\hline & 0.050808 & & \\
\hline & 0 & & \\
\hline \multicolumn{4}{|c|}{ 2-thioformylpyridinium cation } \\
\hline Atomic \# & $\mathrm{X}$ & $\mathrm{Y}$ & $\mathrm{Z}$ \\
\hline 6 & 1.443692 & 1.399243 & 0 \\
\hline 6 & 2.520093 & 0.534265 & 0 \\
\hline 6 & 2.277645 & -0.831708 & 0 \\
\hline 6 & 0.971276 & -1.309569 & 0 \\
\hline 6 & -0.078615 & -0.412066 & 0 \\
\hline 7 & 0.204858 & 0.904776 & 0 \\
\hline
\end{tabular}




\begin{tabular}{|c|c|c|c|}
\hline 1 & 3.105126 & -1.527939 & 0 \\
\hline 1 & 1.539359 & 2.475106 & 0 \\
\hline 1 & 3.523437 & 0.932008 & 0 \\
\hline 1 & -0.610155 & 1.525294 & 0 \\
\hline 16 & -2.719887 & 0.224896 & 0 \\
\hline 6 & -1.496129 & -0.812862 & 0 \\
\hline 1 & 0.760066 & -2.369377 & 0 \\
\hline \multirow[t]{5}{*}{1} & -1.653454 & -1.888457 & 0 \\
\hline & -684.910627 & & \\
\hline & 0.110223 & & \\
\hline & 0.117637 & & \\
\hline & 0 & & \\
\hline \multicolumn{4}{|c|}{$\begin{array}{l}\text { Addition/1,3-butadiene } \\
\text { elimination reaction of 2-pyridyl } \\
\text { cation }\end{array}$} \\
\hline \multicolumn{4}{|c|}{ THF addition product ( 2 position) } \\
\hline Atomic \# & $\mathrm{X}$ & $\mathrm{Y}$ & $\mathrm{Z}$ \\
\hline 6 & $\begin{array}{l}-0.50968 \\
\end{array}$ & -0.326267 & \begin{tabular}{|l}
0.195946 \\
\end{tabular} \\
\hline 6 & -1.510144 & -1.269506 & 0.161635 \\
\hline 6 & -2.787544 & -0.764028 & -0.04251 \\
\hline 6 & -2.961325 & 0.605023 & -0.186436 \\
\hline 6 & -1.853101 & 1.436032 & -0.122221 \\
\hline 7 & -0.617703 & 0.961213 & 0.066483 \\
\hline 1 & -3.634115 & -1.434984 & -0.079187 \\
\hline 1 & -1.306939 & -2.321427 & 0.295978 \\
\hline 1 & -3.943154 & 1.027017 & -0.341282 \\
\hline 1 & -1.944302 & 2.508656 & -0.223166 \\
\hline 6 & 1.720547 & 0.02421 & 1.270266 \\
\hline 8 & 0.837998 & -0.813531 & 0.39264 \\
\hline 6 & 1.616795 & -1.010957 & -0.873473 \\
\hline 6 & 2.271209 & 0.331995 & -1.098928 \\
\hline 6 & 2.554682 & 0.875862 & 0.318391 \\
\hline 1 & 1.056186 & 0.570865 & 1.927762 \\
\hline 1 & 2.294586 & -0.715145 & 1.820147 \\
\hline 1 & 2.314578 & -1.80737 & -0.62784 \\
\hline 1 & 0.9012 & -1.338805 & -1.620334 \\
\hline 1 & 3.177233 & 0.216549 & -1.688092 \\
\hline 1 & 1.593546 & 0.995074 & -1.629512 \\
\hline 1 & 3.606449 & 0.788064 & 0.577391 \\
\hline 1 & 2.265623 & 1.919847 & 0.386636 \\
\hline
\end{tabular}




\begin{tabular}{|c|c|c|c|}
\hline Elec energy & -479.842609 & & \\
\hline ZPVE & 0.200437 & & \\
\hline Thermal correction to enthalpy & 0.210559 & & \\
\hline Negative frequencies & 0 & & \\
\hline \multicolumn{4}{|l|}{ 1,2-Hydride shift TS } \\
\hline Atomic \# & $\mathrm{X}$ & $\mathrm{Y}$ & $\mathrm{Z}$ \\
\hline 6 & 0.605743 & -0.335967 & -0.323205 \\
\hline 6 & 1.697772 & -1.188412 & -0.34717 \\
\hline 6 & 2.932199 & -0.64588 & -0.035694 \\
\hline 6 & 3.019368 & 0.703805 & 0.278924 \\
\hline 6 & 1.862612 & 1.460801 & 0.267925 \\
\hline 7 & 0.662885 & 0.947485 & -0.027212 \\
\hline 1 & 3.816785 & -1.267314 & -0.044834 \\
\hline 1 & 1.571058 & -2.227015 & -0.61512 \\
\hline 1 & 3.965506 & 1.162899 & 0.523008 \\
\hline 1 & 1.886602 & 2.517033 & 0.501207 \\
\hline 6 & -2.224222 & 1.188475 & -0.942607 \\
\hline 8 & -0.642833 & -0.831385 & -0.660693 \\
\hline 6 & -1.402046 & -1.352713 & 0.422612 \\
\hline 6 & -2.220747 & -0.231604 & 1.089228 \\
\hline 6 & -2.967017 & 0.430487 & -0.037932 \\
\hline 1 & -1.269078 & 1.607264 & -0.620646 \\
\hline 1 & -2.635808 & 1.465265 & -1.909093 \\
\hline 1 & -2.057559 & -2.113277 & 0.002037 \\
\hline 1 & -0.746695 & -1.817188 & 1.160046 \\
\hline 1 & -2.897246 & -0.644432 & 1.833062 \\
\hline 1 & -1.544965 & 0.474168 & 1.5698 \\
\hline 1 & -3.87618 & -0.035718 & -0.407048 \\
\hline \multirow[t]{5}{*}{1} & -3.264886 & 1.547823 & \begin{tabular}{|l|}
0.210137 \\
\end{tabular} \\
\hline & -479.789899 & & \\
\hline & 0.193612 & & \\
\hline & 0.204176 & & \\
\hline & 0 & & \\
\hline \multicolumn{4}{|l|}{ Secondary carbocation } \\
\hline Atomic \# & $\mathrm{X}$ & $\mathrm{Y}$ & Z \\
\hline 6 & 0.662433 & -0.203806 & 0.425714 \\
\hline 6 & 1.510488 & -1.297282 & 0.350874 \\
\hline 6 & 2.778621 & -1.05587 & -0.141478 \\
\hline
\end{tabular}




\begin{tabular}{|c|c|c|c|}
\hline 6 & 3.12895 & 0.234322 & -0.528106 \\
\hline 6 & 2.188461 & 1.237813 & -0.408533 \\
\hline 7 & 0.953905 & 1.01876 & 0.064934 \\
\hline 1 & 3.491324 & -1.864916 & -0.219157 \\
\hline 1 & \begin{tabular}{|l|}
1.183891 \\
\end{tabular} & -2.272617 & 0.678795 \\
\hline 1 & 4.112954 & 0.455184 & -0.91296 \\
\hline 1 & \begin{tabular}{|l|}
2.413716 \\
\end{tabular} & 2.255807 & -0.696204 \\
\hline 6 & -2.733627 & -1.24928 & -0.868178 \\
\hline 8 & -0.609935 & -0.444174 & 0.937899 \\
\hline 6 & -1.451172 & 0.605678 & 1.077371 \\
\hline 6 & -2.097859 & 1.157678 & -0.449755 \\
\hline 6 & -3.000374 & 0.106851 & -0.39393 \\
\hline 1 & -1.751403 & -1.362151 & -1.315348 \\
\hline 1 & -2.887194 & -1.972791 & -0.062929 \\
\hline 1 & -2.22855 & 0.366327 & 1.795419 \\
\hline 1 & -0.960959 & 1.55251 & 1.300816 \\
\hline 1 & -2.463691 & 2.157948 & -0.25583 \\
\hline 1 & -1.234952 & 1.08904 & -1.10149 \\
\hline 1 & -3.954752 & 0.282363 & 0.096964 \\
\hline \multirow[t]{5}{*}{1} & -3.508996 & -1.474842 & -1.612407 \\
\hline & -479.811285 & & \\
\hline & \begin{tabular}{|l|l|}
0.194454 \\
\end{tabular} & & \\
\hline & 0.206208 & & \\
\hline & 0 & & \\
\hline \multicolumn{4}{|c|}{ Nitrogen protonated species } \\
\hline Atomic \# & $\mathrm{X}$ & $\mathrm{Y}$ & $Z$ \\
\hline 6 & \begin{tabular}{|l|}
0.478408 \\
\end{tabular} & -0.449542 & -0.465211 \\
\hline 6 & \begin{tabular}{|l|}
0.909399 \\
\end{tabular} & -0.916093 & 0.777777 \\
\hline 6 & \begin{tabular}{|l|}
2.135389 \\
\end{tabular} & -0.502954 & 1.248926 \\
\hline 6 & 2.944516 & 0.370178 & 0.511223 \\
\hline 6 & 2.489462 & 0.803715 & -0.698675 \\
\hline 7 & 1.283548 & 0.389701 & -1.145875 \\
\hline 1 & \begin{tabular}{|l|}
2.479383 \\
\end{tabular} & -0.866105 & 2.207933 \\
\hline 1 & 0.291105 & -1.596258 & 1.340736 \\
\hline 1 & \begin{tabular}{|l|}
3.905307 \\
\end{tabular} & 0.696229 & 0.876154 \\
\hline 1 & \begin{tabular}{|l|}
3.032617 \\
\end{tabular} & 1.473672 & -1.347616 \\
\hline 6 & \begin{tabular}{|l|}
-2.087128 \\
\end{tabular} & 2.006465 & 0.58716 \\
\hline 8 & \begin{tabular}{|l|}
-0.62043 \\
\end{tabular} & -0.74345 & -1.103943 \\
\hline 6 & -1.722284 & -1.386963 & -0.410434 \\
\hline 6 & -2.330236 & -0.471487 & 0.64069 \\
\hline
\end{tabular}




\begin{tabular}{|c|c|c|c|}
\hline 6 & -2.613057 & \begin{tabular}{|l|}
0.895952 \\
\end{tabular} & 0.095104 \\
\hline 1 & 0.932622 & 0.716674 & -2.040339 \\
\hline 1 & -2.323609 & 2.976689 & 0.173262 \\
\hline 1 & -2.425105 & -1.590814 & -1.212492 \\
\hline 1 & -1.375784 & -2.335243 & -0.000075 \\
\hline 1 & -3.253263 & -0.954479 & 0.970873 \\
\hline 1 & -1.685751 & -0.391366 & 1.517019 \\
\hline 1 & -3.28781 & 0.947938 & -0.753896 \\
\hline \multirow[t]{5}{*}{1} & -1.426068 & 1.984127 & 1.446629 \\
\hline & \begin{tabular}{|l}
-479.870065 \\
\end{tabular} & & \\
\hline & \begin{tabular}{|l|}
0.19857 \\
\end{tabular} & & \\
\hline & 0.209658 & & \\
\hline & 0 & & \\
\hline \multicolumn{4}{|c|}{ O proton transfer TS } \\
\hline Atomic \# & $\mathrm{X}$ & $\mathrm{Y}$ & Z \\
\hline 6 & -1.095501 & -0.030425 & 0.099205 \\
\hline 6 & -1.8705 & 1.134065 & -0.161068 \\
\hline 6 & -3.224566 & 1.047911 & -0.296242 \\
\hline 6 & -3.896405 & -0.189306 & -0.183774 \\
\hline 6 & -3.15392 & -1.295699 & 0.063358 \\
\hline 7 & -1.804836 & -1.195823 & 0.196015 \\
\hline 1 & $\mid-3.7966$ & 1.944562 & -0.49361 \\
\hline 1 & -1.349596 & 2.075837 & -0.246096 \\
\hline 1 & -4.966265 & -0.264255 & -0.289551 \\
\hline 1 & -3.56673 & -2.287651 & 0.166849 \\
\hline 6 & \begin{tabular}{|l|}
4.99499 \\
\end{tabular} & -0.766998 & -0.308461 \\
\hline 8 & 0.152371 & -0.07133 & 0.242539 \\
\hline 6 & 1.97161 & 1.28916 & 0.292188 \\
\hline 6 & 2.975522 & 0.34528 & 0.531922 \\
\hline 6 & 3.830386 & -0.190329 & -0.555343 \\
\hline 1 & \begin{tabular}{|l|}
-1.257479 \\
\end{tabular} & -2.026293 & 0.37947 \\
\hline 1 & 5.614584 & -1.138336 & -1.111899 \\
\hline 1 & 1.673211 & 1.545772 & -0.715196 \\
\hline 1 & \begin{tabular}{|l|}
1.477388 \\
\end{tabular} & \begin{tabular}{|l|}
1.79763 \\
\end{tabular} & 1.10714 \\
\hline 1 & 3.372094 & 0.307235 & 1.542623 \\
\hline 1 & \begin{tabular}{|l|}
1.997653 \\
\end{tabular} & -0.304676 & 0.593815 \\
\hline 1 & 3.458201 & -0.083541 & -1.567573 \\
\hline 1 & 5.371225 & -0.88077 & 0.700647 \\
\hline
\end{tabular}




\begin{tabular}{|c|c|c|c|}
\hline & -479.793756 & & \\
\hline & 0.190793 & & \\
\hline & 0.202953 & & \\
\hline & 1 & & \\
\hline \multicolumn{4}{|c|}{ 2-hydroxypyridinium cation (Cs) } \\
\hline Atomic \# & $\mathrm{X}$ & $\mathrm{Y}$ & $\mathrm{Z}$ \\
\hline 6 & 0.027499 & -0.909753 & 0 \\
\hline 6 & 1.24083 & -0.231779 & 0 \\
\hline 6 & 1.223561 & 1.145817 & 0 \\
\hline 6 & 0.015531 & 1.853635 & 0 \\
\hline 6 & -1.151756 & 1.147907 & 0 \\
\hline 7 & -1.114467 & -0.204816 & 0 \\
\hline 1 & 2.16082 & 1.685467 & 0 \\
\hline 1 & 2.165142 & -0.79123 & 0 \\
\hline 1 & -0.007141 & 2.931891 & 0 \\
\hline 1 & -2.133813 & 1.595708 & 0 \\
\hline 1 & -1.979978 & -0.736354 & 0 \\
\hline 8 & -0.157578 & -2.213439 & 0 \\
\hline \multirow[t]{5}{*}{1} & 0.669457 & -2.71211 & 0 \\
\hline & -323.870582 & & \\
\hline & 0.107746 & & \\
\hline & 0.114066 & & \\
\hline & 0 & & \\
\hline \multicolumn{4}{|c|}{ 1,3-butadiene (C2h) } \\
\hline Atomic \# & $\mathrm{X}$ & $\mathrm{Y}$ & $\mathrm{Z}$ \\
\hline 6 & -1.832809 & 0.120137 & 0 \\
\hline 6 & -0.609378 & -0.399459 & 0 \\
\hline 1 & -1.983048 & 1.192949 & 0 \\
\hline 1 & -2.715667 & -0.502924 & 0 \\
\hline 1 & -0.479982 & -1.477127 & 0 \\
\hline 6 & 0.609378 & 0.399459 & 0 \\
\hline 6 & 1.832809 & -0.120137 & 0 \\
\hline 1 & 0.479982 & 1.477127 & 0 \\
\hline 1 & 2.715667 & 0.502924 & 0 \\
\hline \multirow[t]{3}{*}{1} & 1.983048 & -1.192949 & 0 \\
\hline & -155.969178 & & \\
\hline & 0.085612 & & \\
\hline
\end{tabular}




\begin{tabular}{|c|c|c|c|}
\hline & 0.091209 & & \\
\hline & 0 & & \\
\hline \multicolumn{4}{|l|}{$\begin{array}{l}\text { Addition/propylene elimination } \\
\text { reaction of } 3,4- \\
\text { didehydropyridinium cation }\end{array}$} \\
\hline \multicolumn{4}{|l|}{ THF (Cs) } \\
\hline Atomic \# & $\mathrm{X}$ & $\mathrm{Y}$ & $\mathrm{Z}$ \\
\hline 6 & 0.159603 & 0.468803 & -1.124838 \\
\hline 8 & -0.300603 & 1.192636 & 0 \\
\hline 6 & 0.159603 & 0.468803 & 1.124838 \\
\hline 6 & -0.050478 & -1.010276 & 0.772687 \\
\hline 6 & -0.050478 & -1.010276 & -0.772687 \\
\hline 1 & -0.401119 & 0.801199 & -1.995019 \\
\hline 1 & 1.223618 & 0.67873 & -1.288273 \\
\hline 1 & 1.223618 & 0.67873 & 1.288273 \\
\hline 1 & -0.401119 & 0.801199 & 1.995019 \\
\hline 1 & 0.732454 & -1.638755 & 1.191446 \\
\hline 1 & -1.004726 & -1.363055 & 1.157503 \\
\hline 1 & 0.732454 & -1.638755 & -1.191446 \\
\hline 1 & -1.004726 & -1.363055 & -1.157503 \\
\hline Elec energy & -232.431693 & & \\
\hline ZPVE & 0.118003 & & \\
\hline Thermal correction to enthalpy & 0.123829 & & \\
\hline Negative frequencies & 0 & & \\
\hline \multicolumn{4}{|l|}{ Addition intermediate } \\
\hline Atomic \# & $\mathrm{X}$ & $\mathrm{Y}$ & $\mathrm{Z}$ \\
\hline 6 & 0.833457 & 1.12939 & -0.228788 \\
\hline 6 & 2.204639 & 1.323686 & -0.340314 \\
\hline 7 & 3.105827 & 0.333392 & -0.140604 \\
\hline 6 & 2.765766 & -0.924764 & \begin{tabular}{|l}
0.185914 \\
\end{tabular} \\
\hline 6 & 1.43181 & -1.224968 & 0.3241 \\
\hline 6 & 0.569248 & -0.162555 & \begin{tabular}{|l}
0.102717 \\
\end{tabular} \\
\hline 6 & -1.584822 & -0.602873 & -1.059148 \\
\hline 6 & -1.651756 & 0.310295 & 1.209592 \\
\hline 6 & -3.006893 & -0.299291 & -0.661006 \\
\hline 6 & -2.823931 & 0.798974 & 0.389006 \\
\hline 1 & 2.65682 & 2.274612 & -0.595783 \\
\hline 1 & 3.56445 & -1.635989 & 0.325567 \\
\hline 1 & 1.109341 & -2.220822 & 0.591051 \\
\hline
\end{tabular}




\begin{tabular}{|l|l|l|l|}
\hline 1 & -1.148258 & 0.173044 & -1.68513 \\
\hline 1 & -1.389765 & -1.597279 & -1.447624 \\
\hline 1 & -1.920782 & -0.381674 & 2.001861 \\
\hline 1 & -0.982382 & 1.090093 & 1.554865 \\
\hline 1 & -3.584338 & 0.034012 & -1.519571 \\
\hline 1 & -3.487783 & -1.178983 & -0.235521 \\
\hline 1 & -3.704842 & 0.938172 & 1.010003 \\
\hline 1 & -2.570731 & 1.743569 & -0.089639 \\
\hline 1 & 4.091674 & 0.548196 & -0.240244 \\
\hline 8 & -0.856215 & -0.529447 & 0.244818 \\
\hline & & & \\
\hline & -479.773657 & & \\
\hline & 0.200165 & & \\
\hline & 0.210434 & & \\
\hline & 0 & & \\
\hline & & & \\
\hline Ring closure TS & & & \\
\hline Atomic \# & $\mathrm{X}$ & $\mathrm{Y}$ & $\mathrm{Z}$ \\
\hline 6 & 0.631777 & 0.993574 & 0.310206 \\
\hline 6 & 1.913464 & 1.371509 & -0.062155 \\
\hline 7 & 2.880919 & 0.456004 & -0.337378 \\
\hline 6 & 2.671451 & -0.872855 & -0.29115 \\
\hline 6 & 1.421557 & -1.34414 & 0.058245 \\
\hline 6 & 0.453881 & -0.380462 & 0.360225 \\
\hline 6 & -1.691085 & -1.282618 & -0.229073 \\
\hline 6 & -1.791156 & 1.003806 & 1.151313 \\
\hline 6 & -2.101313 & -0.103421 & -1.090722 \\
\hline 6 & -2.465518 & 1.08764 & -0.17096 \\
\hline 1 & 2.238494 & 2.40902 & -0.163021 \\
\hline 1 & 3.515883 & -1.517602 & -0.526674 \\
\hline 1 & 1.233634 & -2.415939 & 0.116218 \\
\hline 1 & -1.183204 & -2.072997 & -0.80032 \\
\hline 1 & -2.528851 & -1.727508 & 0.322346 \\
\hline 1 & -2.233038 & 0.364012 & 1.917967 \\
\hline 1 & -1.217555 & 1.848514 & 1.520642 \\
\hline 1 & -1.256933 & 0.199382 & -1.725811 \\
\hline 1 & -2.93526 & -0.379118 & -1.748444 \\
\hline 1 & -3.549795 & 1.065876 & 0.035925 \\
\hline 1 & -2.20678 & 2.031357 & -0.665002 \\
\hline 1 & 3.811154 & 0.786532 & -0.590665 \\
\hline 8 & -0.795958 & -0.785689 & 0.794728 \\
\hline & & & \\
\hline 1 & & & \\
\hline & & & \\
\hline & & & \\
\hline & & & \\
\hline & & & \\
\hline & & & \\
\hline & & & \\
\hline & & & \\
\hline
\end{tabular}




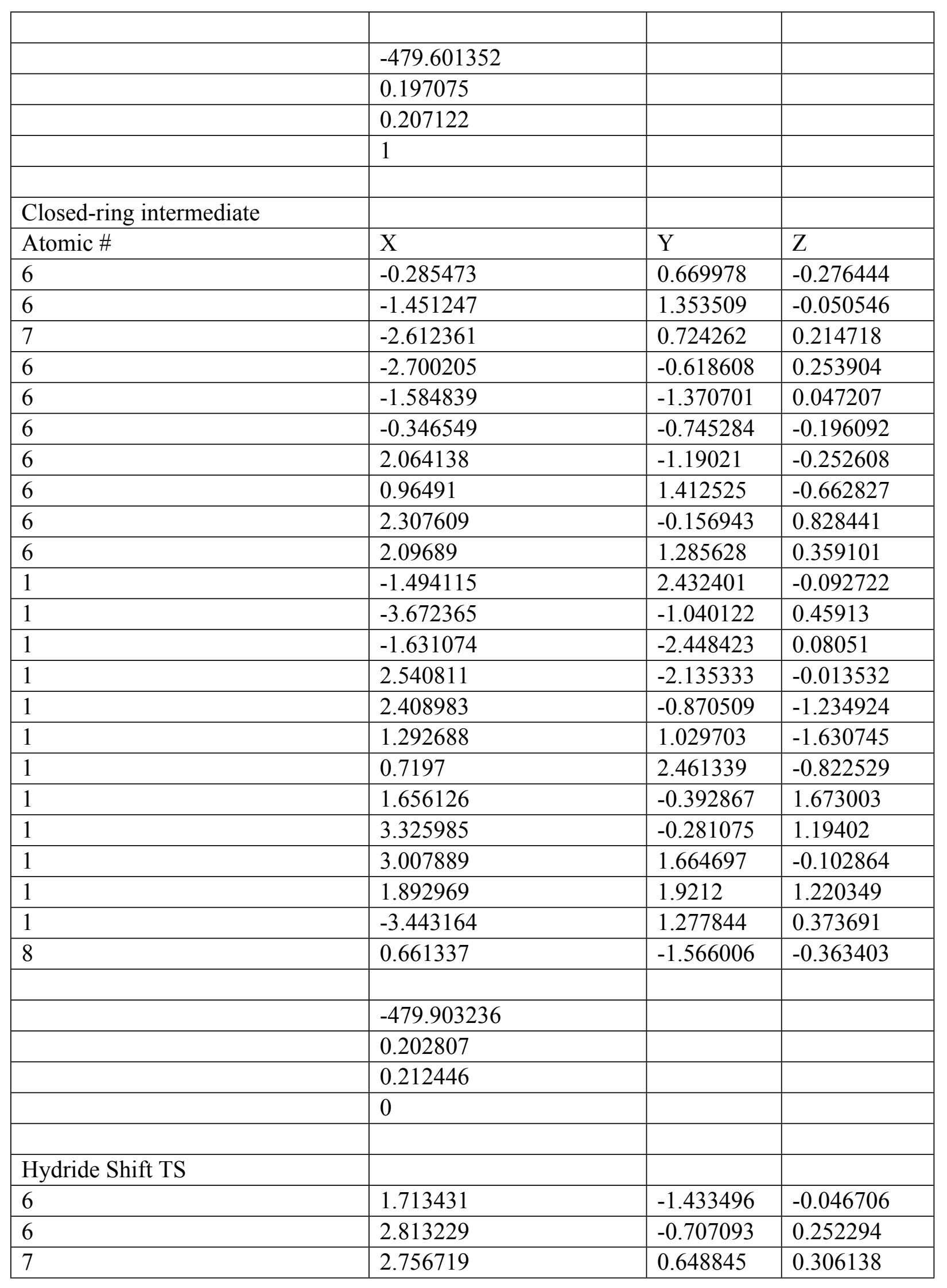




\begin{tabular}{|c|c|c|c|}
\hline 6 & 1.601224 & 1.31314 & 0.055316 \\
\hline 6 & 0.450842 & 0.659776 & -0.244947 \\
\hline 6 & 0.450019 & -0.793868 & -0.311045 \\
\hline 6 & -0.798614 & 1.431741 & -0.572764 \\
\hline 6 & -1.979908 & 1.247301 & 0.421659 \\
\hline 6 & -2.788773 & 0.074026 & -0.017455 \\
\hline 6 & -2.670607 & -1.18043 & 0.530649 \\
\hline 8 & -0.581266 & -1.434809 & -0.581534 \\
\hline 1 & 1.765778 & -2.510267 & -0.09855 \\
\hline 1 & 3.776276 & -1.150325 & 0.457851 \\
\hline 1 & 1.661666 & 2.391861 & 0.101323 \\
\hline 1 & \begin{tabular}{|l}
-0.565599 \\
\end{tabular} & 2.494605 & -0.596823 \\
\hline 1 & -1.128234 & 1.160322 & -1.579206 \\
\hline 1 & -1.604281 & 1.102733 & 1.434068 \\
\hline 1 & -2.599915 & 2.141777 & 0.402302 \\
\hline 1 & -3.656818 & -0.341724 & 0.87812 \\
\hline 1 & -3.382517 & 0.167553 & -0.922261 \\
\hline 1 & -2.082083 & -1.327015 & 1.426663 \\
\hline 1 & -3.165886 & -2.031444 & 0.080706 \\
\hline \multirow[t]{5}{*}{1} & 3.58965 & 1.171899 & 0.523104 \\
\hline & -479.814319 & & \\
\hline & 0.194557 & & \\
\hline & 0.205171 & & \\
\hline & 1 & & \\
\hline \multicolumn{4}{|c|}{ 6-membered ring intermediate } \\
\hline & & & \\
\hline 6 & \begin{tabular}{|l|}
1.339007 \\
\end{tabular} & -1.524261 & 0.030797 \\
\hline 6 & 2.62678 & -1.087246 & 0.089705 \\
\hline 7 & \begin{tabular}{|l|}
2.888421 \\
\end{tabular} & 0.235429 & 0.064128 \\
\hline 6 & \begin{tabular}{|l|}
1.909981 \\
\end{tabular} & 1.158112 & -0.01263 \\
\hline 6 & 0.592468 & 0.797975 & -0.08699 \\
\hline 6 & \begin{tabular}{|l|}
0.296289 \\
\end{tabular} & -0.584982 & -0.069559 \\
\hline 6 & -0.523275 & 1.803885 & -0.14707 \\
\hline 6 & -1.804309 & 1.160647 & 0.373048 \\
\hline 6 & -2.056051 & -0.154052 & -0.336148 \\
\hline 6 & -3.272777 & -0.897566 & 0.152065 \\
\hline 8 & -0.922007 & -1.057681 & -0.131765 \\
\hline 1 & 1.108397 & -2.578408 & 0.05235 \\
\hline 1 & \begin{tabular}{|l|}
3.476178 \\
\end{tabular} & -1.749624 & 0.15777 \\
\hline
\end{tabular}




\begin{tabular}{|c|c|c|c|}
\hline 1 & 2.224628 & 2.192224 & -0.008194 \\
\hline 1 & -0.258958 & 2.6874 & 0.432083 \\
\hline 1 & -0.66469 & 2.127341 & -1.180593 \\
\hline 1 & -1.734496 & 0.977812 & 1.447564 \\
\hline 1 & -2.656849 & 1.816097 & 0.205406 \\
\hline 1 & -4.164308 & -0.306991 & -0.052248 \\
\hline 1 & -2.107789 & 0.005398 & -1.415851 \\
\hline 1 & -3.206534 & -1.071333 & 1.225349 \\
\hline 1 & -3.367084 & -1.854542 & -0.356093 \\
\hline \multirow[t]{5}{*}{1} & 3.849212 & 0.546081 & 0.111816 \\
\hline & -479.918348 & & \\
\hline & 0.201399 & & \\
\hline & 0.211342 & & \\
\hline & 0 & & \\
\hline \multicolumn{4}{|c|}{ Ketene product $(\mathrm{Cs})$} \\
\hline Atomic \# & $\mathrm{X}$ & $\mathrm{Y}$ & $\mathrm{Z}$ \\
\hline 6 & 0.505098 & -1.504244 & 0 \\
\hline 6 & -0.806274 & -1.730195 & 0 \\
\hline 7 & -1.729991 & -0.658125 & 0 \\
\hline 6 & -1.377956 & 0.591936 & 0 \\
\hline 6 & 0.000617 & 0.955694 & 0 \\
\hline 6 & 1.042696 & -0.12919 & 0 \\
\hline 8 & 2.215813 & 0.122352 & 0 \\
\hline 6 & 0.351282 & 2.25538 & 0 \\
\hline 1 & 1.209491 & -2.323669 & 0 \\
\hline 1 & -1.259805 & -2.709387 & 0 \\
\hline 1 & -2.16417 & 1.337825 & 0 \\
\hline 1 & 1.402039 & 2.522904 & 0 \\
\hline 1 & -0.386078 & 3.048704 & 0 \\
\hline \multirow[t]{5}{*}{1} & -2.716806 & -0.891046 & 0 \\
\hline & 261005510 & & \\
\hline & 0.111595 & & \\
\hline & 0.118984 & & \\
\hline & 0 & & \\
\hline \multicolumn{4}{|c|}{ Propene (Cs) } \\
\hline Atomic \# & $\mathrm{X}$ & $\mathrm{Y}$ & $Z$ \\
\hline 6 & 1.271145 & -0.219391 & 0 \\
\hline
\end{tabular}




\begin{tabular}{|c|c|c|c|}
\hline 6 & 0.132779 & 0.456652 & 0 \\
\hline 6 & -1.228321 & -0.16284 & 0 \\
\hline 1 & 2.228641 & 0.283063 & 0 \\
\hline 1 & \begin{tabular}{|l|}
1.280571 \\
\end{tabular} & -1.303013 & 0 \\
\hline 1 & 0.167952 & 1.541749 & 0 \\
\hline 1 & -1.7982 & 0.149849 & -0.876573 \\
\hline 1 & -1.164429 & -1.249947 & 0 \\
\hline \multirow[t]{5}{*}{1} & -1.7982 & 0.149849 & 0.876573 \\
\hline & -117.885254 & & \\
\hline & 0.080112 & & \\
\hline & 0.085142 & & \\
\hline & 0 & & \\
\hline \multicolumn{4}{|l|}{$\begin{array}{l}\text { Addition/acetylene elimination } \\
\text { reaction of } 3,4- \\
\text { didehydropyridinium cation }\end{array}$} \\
\hline \multicolumn{4}{|l|}{ Furan $(\mathrm{C} 2 \mathrm{v})$} \\
\hline Atomic \# & $\mathrm{X}$ & $\mathrm{Y}$ & $\mathrm{Z}$ \\
\hline 6 & 0 & -1.087433 & -0.336025 \\
\hline 6 & 0 & -0.716291 & 0.963169 \\
\hline 6 & 0 & 0.716291 & 0.963169 \\
\hline 6 & 0 & 1.087433 & -0.336025 \\
\hline 8 & 0 & 0 & -1.140722 \\
\hline 1 & 0 & -2.04138 & -0.831176 \\
\hline 1 & 0 & -1.372654 & 1.815205 \\
\hline 1 & 0 & 1.372654 & 1.815205 \\
\hline 1 & 0 & 2.04138 & -0.831176 \\
\hline Elec energy & -230.015871 & & \\
\hline ZPVE & 0.070885 & & \\
\hline Thermal correction to enthalpy & 0.075482 & & \\
\hline Negative frequencies & 0 & & \\
\hline \multicolumn{4}{|l|}{ Diels-Alder product } \\
\hline Atomic \# & $\mathrm{X}$ & $\mathrm{Y}$ & $\mathrm{Z}$ \\
\hline 6 & 1.352209 & -1.374493 & 0.006502 \\
\hline 6 & 0.184082 & -0.700527 & -0.14383 \\
\hline 6 & 0.179143 & 0.704615 & -0.147843 \\
\hline 6 & 1.340615 & 1.425217 & 0.00811 \\
\hline 6 & 2.512529 & 0.699621 & 0.155698 \\
\hline 7 & 2.487219 & -0.635991 & 0.15074 \\
\hline 1 & 1.468522 & -2.447751 & 0.014312 \\
\hline 1 & 1.37452 & 2.504496 & 0.00495 \\
\hline
\end{tabular}




\begin{tabular}{|c|c|c|c|}
\hline 1 & 3.482359 & 1.160702 & 0.271181 \\
\hline 1 & 3.366211 & -1.129032 & \begin{tabular}{|l|}
0.251273 \\
\end{tabular} \\
\hline 6 & -1.294957 & 1.064247 & -0.361338 \\
\hline 6 & -1.996479 & 0.664103 & 0.944206 \\
\hline 6 & -1.998229 & -0.659829 & 0.95033 \\
\hline 6 & -1.289754 & -1.077417 & -0.345732 \\
\hline 8 & -1.655094 & -0.0136 & -1.221075 \\
\hline 1 & -1.513097 & 2.030916 & -0.797702 \\
\hline 1 & -2.356811 & 1.354126 & 1.689408 \\
\hline 1 & -2.366029 & -1.339198 & 1.701878 \\
\hline \multirow[t]{5}{*}{1} & -1.505304 & -2.049523 & -0.770726 \\
\hline & -477.425748 & & \\
\hline & 0.155593 & & \\
\hline & 0.163588 & & \\
\hline & 0 & & \\
\hline \multicolumn{4}{|l|}{ Acetylene loss TS } \\
\hline Atomic \# & $X$ & $\mathrm{Y}$ & $\mathrm{Z}$ \\
\hline 6 & 1.28696 & -1.386697 & -0.093921 \\
\hline 6 & 0.145496 & -0.679314 & 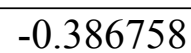 \\
\hline 6 & 0.16072 & 0.746565 & -0.340329 \\
\hline 6 & 1.341943 & 1.422903 & \begin{tabular}{|l|}
0.015358 \\
\end{tabular} \\
\hline 6 & 2.444989 & 0.670957 & 0.271676 \\
\hline 7 & 2.389174 & -0.694963 & 0.208206 \\
\hline 1 & 1.363957 & -2.46422 & -0.093024 \\
\hline 1 & 1.40021 & 2.499404 & 0.070643 \\
\hline 1 & 3.406773 & 1.088097 & \begin{tabular}{|l|}
0.524578 \\
\end{tabular} \\
\hline 1 & 3.23962 & -1.209211 & \begin{tabular}{|l|}
0.396852 \\
\end{tabular} \\
\hline 6 & -1.145912 & 1.135856 & $\begin{array}{l}-0.662879 \\
\end{array}$ \\
\hline 6 & -1.849441 & 0.539612 & 1.437131 \\
\hline 6 & -1.913317 & -0.680812 & 1.306605 \\
\hline 6 & -1.205185 & -1.030735 & -0.671976 \\
\hline 8 & -1.802239 & 0.067844 & -1.15767 \\
\hline 1 & -1.549882 & 2.104631 & -0.901477 \\
\hline 1 & -1.94872 & 1.506475 & \begin{tabular}{|l|}
1.878544 \\
\end{tabular} \\
\hline 1 & -2.160849 & -1.664824 & 1.644226 \\
\hline \multirow[t]{3}{*}{1} & -1.606083 & -1.975966 & -0.999101 \\
\hline & -477.348449 & & \\
\hline & 0.149707 & & \\
\hline
\end{tabular}




\begin{tabular}{|c|c|c|c|}
\hline & 0.158781 & & \\
\hline & 1 & & \\
\hline \multicolumn{4}{|l|}{ Acetylene (dinfh) } \\
\hline Atomic \# & $\mathrm{X}$ & $\mathrm{Y}$ & $\mathrm{Z}$ \\
\hline 6 & 0 & 0 & -0.59695 \\
\hline 6 & 0 & 0 & 0.59695 \\
\hline 1 & 0 & 0 & -1.659875 \\
\hline \multirow[t]{5}{*}{1} & 0 & 0 & 1.659875 \\
\hline & -77.32531 & & \\
\hline & 0.027469 & & \\
\hline & 0.031155 & & \\
\hline & 0 & & \\
\hline \multicolumn{4}{|l|}{ furo[3,4-c]pyridinium } \\
\hline Atomic \# & $\mathrm{X}$ & $\mathrm{Y}$ & $Z$ \\
\hline 6 & 1.297587 & -1.392009 & -0.110299 \\
\hline 6 & 0.143075 & -0.677042 & -0.426885 \\
\hline 6 & 0.158649 & 0.76977 & -0.383668 \\
\hline 6 & 1.363316 & 1.435414 & -0.015536 \\
\hline 6 & 2.440573 & 0.682474 & \begin{tabular}{|l|}
0.277484 \\
\end{tabular} \\
\hline 7 & 2.372457 & -0.708879 & 0.220506 \\
\hline 1 & 1.369305 & -2.470913 & \begin{tabular}{|l|}
-0.118348 \\
\end{tabular} \\
\hline 1 & 1.430876 & 2.511943 & 0.032913 \\
\hline 1 & 3.398982 & 1.081331 & 0.56678 \\
\hline 1 & 3.213879 & -1.222669 & 0.451458 \\
\hline 6 & -1.096664 & 1.15801 & -0.738416 \\
\hline 6 & -1.130241 & -1.042161 & -0.80692 \\
\hline 8 & -1.841663 & 0.056139 & \begin{tabular}{|l|}
-0.984539 \\
\end{tabular} \\
\hline 1 & -1.589826 & 2.105442 & -0.856583 \\
\hline \multirow[t]{5}{*}{1} & -1.621349 & -1.984884 & -0.975292 \\
\hline & -400.04339 & & \\
\hline & 0.120545 & & \\
\hline & 0.127417 & & \\
\hline & 0 & & \\
\hline \multicolumn{4}{|l|}{\begin{tabular}{|l|} 
Addition/CO elimination \\
reaction of 2-pyridyl cation
\end{tabular}} \\
\hline \multicolumn{4}{|l|}{ Oxygen-bound adduct } \\
\hline Atomic \# & $\mathrm{X}$ & $\mathrm{Y}$ & $\mathrm{Z}$ \\
\hline
\end{tabular}




\begin{tabular}{|c|c|c|c|}
\hline 6 & 0.509978 & 0.030905 & 0.017721 \\
\hline 6 & \begin{tabular}{|l|}
1.159943 \\
\end{tabular} & 1.20233 & 0.319639 \\
\hline 6 & 2.545616 & 1.108716 & 0.26453 \\
\hline 6 & 3.139428 & -0.104322 & -0.054554 \\
\hline 6 & 2.334199 & -1.204279 & -0.307089 \\
\hline 7 & 0.999194 & -1.123707 & -0.275185 \\
\hline 1 & 3.149229 & 1.977992 & 0.483817 \\
\hline 1 & 0.64784 & 2.110737 & 0.598786 \\
\hline 1 & 4.213947 & -0.200739 & -0.09841 \\
\hline 1 & 2.749685 & -2.173203 & -0.545518 \\
\hline 6 & -1.801376 & 1.071617 & -0.422756 \\
\hline 6 & -3.054003 & 0.641883 & -0.286995 \\
\hline 6 & -3.025441 & -0.698503 & 0.256333 \\
\hline 6 & -1.757008 & -1.060868 & 0.435035 \\
\hline 8 & -0.957278 & 0.034099 & 0.032949 \\
\hline 1 & -1.300499 & 1.945984 & -0.794071 \\
\hline 1 & -3.931331 & 1.206076 & -0.553444 \\
\hline 1 & -3.879098 & -1.314185 & 0.481917 \\
\hline 1 & -1.208248 & -1.918762 & 0.775776 \\
\hline Elec energy & -477.381403 & & \\
\hline ZPVE & 0.15118 & & \\
\hline Thermal correction to enthalpy & \begin{tabular}{|l|l|}
0.160607 \\
\end{tabular} & & \\
\hline Negative frequencies & 0 & & \\
\hline \multicolumn{4}{|l|}{$\mathrm{O}->\mathrm{C}$ bound TS } \\
\hline Atomic \# & $X$ & $\mathrm{Y}$ & $\mathrm{Z}$ \\
\hline 6 & 0.497517 & -0.273446 & -0.083928 \\
\hline 6 & \begin{tabular}{|l|}
1.389245 \\
\end{tabular} & -1.306449 & -0.142224 \\
\hline 6 & \begin{tabular}{|l|}
2.679794 \\
\end{tabular} & $\begin{array}{l}-0.799771 \\
\end{array}$ & 0.005673 \\
\hline 6 & \begin{tabular}{|l|}
2.908893 \\
\end{tabular} & 0.569453 & 0.165262 \\
\hline 6 & \begin{tabular}{|l|}
1.843649 \\
\end{tabular} & 1.449164 & 0.170469 \\
\hline 7 & \begin{tabular}{|l|}
0.596526 \\
\end{tabular} & 0.946727 & 0.019446 \\
\hline 1 & 3.511319 & -1.491987 & -0.010483 \\
\hline 1 & \begin{tabular}{|l|}
1.150272 \\
\end{tabular} & -2.348644 & -0.276103 \\
\hline 1 & \begin{tabular}{|l|}
3.915167 \\
\end{tabular} & 0.946664 & 0.270664 \\
\hline 1 & \begin{tabular}{|l|}
1.941829 \\
\end{tabular} & 2.518941 & 0.274758 \\
\hline 6 & -1.530194 & -1.012399 & 0.566266 \\
\hline 6 & \begin{tabular}{|l|}
-2.364662 \\
\end{tabular} & -0.092149 & 1.089906 \\
\hline 6 & \begin{tabular}{|l|}
-2.652108 \\
\end{tabular} & 0.841968 & 0.053957 \\
\hline 6 & -1.984344 & \begin{tabular}{|l|}
0.433437 \\
\end{tabular} & -1.051489 \\
\hline 8 & \begin{tabular}{|l|}
-1.289051 \\
\end{tabular} & -0.696287 & -0.786795 \\
\hline
\end{tabular}




\begin{tabular}{|c|c|c|c|}
\hline 1 & -1.167958 & -1.970953 & 0.890429 \\
\hline 1 & -2.740853 & -0.083772 & 2.098362 \\
\hline 1 & -3.296745 & 1.701932 & 0.118194 \\
\hline \multirow[t]{5}{*}{1} & -1.877795 & 0.832547 & -2.044386 \\
\hline & -477.350353 & & \\
\hline & 0.14931 & & \\
\hline & 0.158511 & & \\
\hline & 1 & & \\
\hline \multicolumn{4}{|c|}{ Carbon-bound adduct } \\
\hline Atomic \# & $X$ & $\mathrm{Y}$ & $\mathrm{Z}$ \\
\hline 6 & \begin{tabular}{|l|}
0.445724 \\
\end{tabular} & -0.385043 & -0.022473 \\
\hline 6 & 1.504503 & -1.268506 & -0.093733 \\
\hline 6 & 2.785768 & -0.729505 & -0.041102 \\
\hline 6 & 2.929743 & 0.640541 & 0.068504 \\
\hline 6 & 1.78784 & 1.435195 & 0.126461 \\
\hline 7 & 0.560537 & 0.930817 & 0.090223 \\
\hline 1 & 3.651106 & -1.375091 & -0.093992 \\
\hline 1 & 1.344761 & -2.332887 & -0.195334 \\
\hline 1 & 3.907477 & 1.097912 & 0.107685 \\
\hline 1 & 1.864433 & 2.511411 & 0.209423 \\
\hline 6 & -0.99655 & -0.88091 & -0.062405 \\
\hline 6 & -1.777525 & -0.458498 & 1.119903 \\
\hline 6 & -2.72029 & 0.430549 & 0.746739 \\
\hline 6 & -2.586495 & 0.541425 & -0.661584 \\
\hline 8 & -1.678004 & -0.20149 & -1.153818 \\
\hline 1 & -1.047708 & -1.950028 & -0.272054 \\
\hline 1 & -1.553752 & -0.802075 & 2.119358 \\
\hline 1 & -3.43164 & 0.96585 & 1.352099 \\
\hline \multirow[t]{5}{*}{1} & -3.151006 & 1.149142 & -1.359316 \\
\hline & -477.42432 & & \\
\hline & 0.152554 & & \\
\hline & 0.161727 & & \\
\hline & 0 & & \\
\hline \multicolumn{4}{|c|}{ Ring opening TS } \\
\hline Atomic \# & $X$ & $\mathrm{Y}$ & Z \\
\hline 6 & 0.618537 & -0.056065 & 0.327075 \\
\hline 6 & 1.424132 & -1.197245 & 0.452703 \\
\hline 6 & 2.759774 & -1.10739 & 0.088077 \\
\hline 6 & 3.218267 & 0.107705 & -0.376187 \\
\hline 6 & 2.335539 & 1.209704 & -0.464848 \\
\hline
\end{tabular}




\begin{tabular}{|c|c|c|c|}
\hline 7 & 1.075019 & 1.138394 & -0.129163 \\
\hline 1 & 3.416987 & -1.961337 & \begin{tabular}{|l|}
0.164624 \\
\end{tabular} \\
\hline 1 & 0.998636 & -2.123242 & 0.816042 \\
\hline 1 & 4.250798 & 0.233717 & -0.672434 \\
\hline 1 & 2.697654 & 2.165949 & -0.821339 \\
\hline 6 & -0.750788 & -0.13265 & 0.651827 \\
\hline 6 & -1.628694 & 1.000729 & \begin{tabular}{|l|}
0.58934 \\
\end{tabular} \\
\hline 6 & -2.876065 & 0.894363 & 0.106868 \\
\hline 6 & -3.274679 & -0.418719 & -0.447738 \\
\hline 8 & -2.431247 & -1.237698 & -0.726595 \\
\hline 1 & -1.121266 & -1.067154 & 1.055621 \\
\hline 1 & -1.215433 & 1.963272 & 0.868277 \\
\hline 1 & -3.561842 & 1.730208 & 0.090265 \\
\hline \multirow[t]{5}{*}{1} & -4.34372 & -0.636952 & -0.567102 \\
\hline & -477.383035 & & \\
\hline & 0.149451 & & \\
\hline & 0.158853 & & \\
\hline & 1 & & \\
\hline \multicolumn{4}{|c|}{ Ring-opened species } \\
\hline Atomic \# & $\mathrm{X}$ & $\mathrm{Y}$ & Z \\
\hline 6 & 0.745925 & 0.075403 & 0.000215 \\
\hline 6 & 1.458033 & 1.288225 & \begin{tabular}{|l|}
0.000291 \\
\end{tabular} \\
\hline 6 & 2.844128 & 1.246465 & \begin{tabular}{|l|}
0.000138 \\
\end{tabular} \\
\hline 6 & 3.447027 & 0.005763 & -0.00008 \\
\hline 6 & 2.653409 & -1.167716 & $\begin{array}{l}-0.000139 \\
\end{array}$ \\
\hline 7 & 1.348552 & -1.142942 & -0.000001 \\
\hline 1 & 3.430319 & 2.153863 & 0.000186 \\
\hline 1 & 0.920726 & 2.2274 & \begin{tabular}{|l|}
0.000458 \\
\end{tabular} \\
\hline 1 & 4.524696 & -0.086987 & -0.000206 \\
\hline 1 & 3.12874 & -2.140752 & -0.000304 \\
\hline 6 & -0.661584 & 0.112311 & \begin{tabular}{|l|}
0.000351 \\
\end{tabular} \\
\hline 6 & -1.464581 & -1.055328 & 0.000282 \\
\hline 6 & -2.817993 & -1.01819 & 0.000155 \\
\hline 6 & -3.700337 & 0.203175 & -0.000034 \\
\hline 8 & -3.279013 & 1.32576 & -0.001143 \\
\hline 1 & -1.152178 & 1.077976 & \begin{tabular}{|l|}
0.000489 \\
\end{tabular} \\
\hline 1 & -0.953739 & -2.008971 & \begin{tabular}{|l|}
0.000339 \\
\end{tabular} \\
\hline 1 & -3.351401 & -1.962885 & 0.000238 \\
\hline \multirow[t]{2}{*}{1} & -4.779089 & -0.005779 & \begin{tabular}{|l|}
0.000872 \\
\end{tabular} \\
\hline & -477.384055 & & \\
\hline
\end{tabular}




\begin{tabular}{|c|c|c|c|}
\hline & 0.149395 & & \\
\hline & 0.159183 & & \\
\hline & 0 & & \\
\hline \multicolumn{4}{|c|}{ Hydride transfer TS } \\
\hline Atomic \# & $\mathrm{X}$ & $\mathrm{Y}$ & $\mathrm{Z}$ \\
\hline 6 & 0.834823 & 0.110112 & -0.034009 \\
\hline 6 & 1.544173 & 1.308101 & -0.074384 \\
\hline 6 & 2.931221 & 1.256691 & -0.024939 \\
\hline 6 & 3.536514 & 0.019738 & 0.060421 \\
\hline 6 & 2.736825 & -1.132494 & 0.094616 \\
\hline 7 & 1.422435 & -1.096572 & 0.049129 \\
\hline 1 & 3.518629 & 2.1632 & -0.053177 \\
\hline 1 & 1.021089 & 2.252392 & -0.143312 \\
\hline 1 & 4.612278 & -0.074242 & 0.101434 \\
\hline 1 & 3.19415 & -2.111597 & 0.161905 \\
\hline 6 & -0.608993 & 0.126208 & -0.084048 \\
\hline 6 & -1.364991 & -1.000781 & -0.087968 \\
\hline 6 & -2.777904 & -0.949552 & -0.217325 \\
\hline 6 & -3.562174 & 0.154993 & 0.099782 \\
\hline 8 & -4.093167 & 1.175695 & 0.084859 \\
\hline 1 & -1.083244 & 1.102712 & -0.138089 \\
\hline 1 & -0.896742 & -1.972926 & -0.036049 \\
\hline 1 & -3.372647 & -1.8163 & -0.497719 \\
\hline \multirow[t]{5}{*}{1} & -3.549497 & -0.693801 & 1.084924 \\
\hline & -477.340576 & & \\
\hline & 0.14511 & & \\
\hline & 0.155243 & & \\
\hline & 1 & & \\
\hline \multicolumn{4}{|c|}{ CO triple bond species } \\
\hline Atomic \# & $\mathrm{X}$ & $\mathrm{Y}$ & $\mathrm{Z}$ \\
\hline 6 & 0.864512 & 0.142225 & -0.180848 \\
\hline 6 & 1.659352 & 1.197661 & -0.607523 \\
\hline 6 & 3.026657 & 1.132561 & -0.372285 \\
\hline 6 & 3.532663 & 0.02225 & 0.273802 \\
\hline 6 & 2.651202 & -0.987533 & 0.664522 \\
\hline 7 & 1.348831 & -0.93597 & 0.446797 \\
\hline 1 & 3.677648 & 1.934899 & -0.689515 \\
\hline 1 & 1.219419 & 2.047272 & -1.111855 \\
\hline 1 & 4.58874 & -0.074466 & 0.479435 \\
\hline 1 & 3.020239 & -1.868707 & 1.174223 \\
\hline 6 & -0.583044 & 0.172616 & -0.413692 \\
\hline
\end{tabular}




\begin{tabular}{|l|l|l|l|}
\hline 6 & -1.38401 & -0.841362 & -0.092426 \\
\hline 6 & -2.874894 & -0.793374 & -0.373597 \\
\hline 6 & -3.484061 & -0.485952 & 0.899095 \\
\hline 8 & -3.858775 & -0.239691 & 1.915841 \\
\hline 1 & -0.981623 & 1.063462 & -0.889685 \\
\hline 1 & -0.99669 & -1.734468 & 0.378811 \\
\hline 1 & -3.170444 & 0.009203 & -1.057066 \\
\hline 1 & -3.306819 & -1.738312 & -0.719125 \\
\hline & -477.404601 & & \\
\hline & 0.149359 & & \\
\hline & 0.159873 & & \\
\hline & 0 & & \\
\hline CO loss TS & & & \\
\hline Atomic \# & $\mathrm{X}$ & $\mathrm{Y}$ & $\mathrm{Z}$ \\
\hline 6 & 0.903604 & 0.300481 & -0.145126 \\
\hline 6 & 1.832013 & 1.260331 & 0.254926 \\
\hline 6 & 3.138343 & 0.8574 & 0.498219 \\
\hline 6 & 3.451077 & -0.475933 & 0.330612 \\
\hline 6 & 2.447765 & -1.370177 & -0.075037 \\
\hline 7 & 1.207732 & -1.000774 & -0.308971 \\
\hline 1 & 3.888024 & 1.570566 & 0.809568 \\
\hline 1 & 1.533957 & 2.293695 & 0.370349 \\
\hline 1 & 4.453149 & -0.840731 & 0.506007 \\
\hline 1 & 2.67635 & -2.419839 & -0.21056 \\
\hline 6 & -0.460644 & 0.686877 & -0.403823 \\
\hline 6 & -1.424222 & -0.170593 & -0.847361 \\
\hline 6 & -2.700212 & 0.322281 & -1.174298 \\
\hline 6 & -3.855935 & -0.061778 & 0.431963 \\
\hline 8 & -4.060965 & -0.43396 & 1.461453 \\
\hline 1 & -0.710785 & 1.733336 & -0.253321 \\
\hline 1 & -1.184187 & -1.214643 & -0.993572 \\
\hline 1 & -2.884865 & 1.390301 & -1.155057 \\
\hline 1 & -3.35024 & -0.242639 & -1.830212 \\
\hline & -477.382803 & & \\
\hline & 0.147463 & & \\
\hline & 0.158326 & & \\
\hline Addn-CO product & 1 & & \\
\hline Atomic \# & & $\mathrm{Y}$ & $\mathrm{Z}$ \\
\hline 6 & $\mathrm{X}$ & 0.734401 & -0.00006 \\
\hline 6 & 0.212311 & 1.411631 & -0.000046 \\
\hline & -0.996884 & & \\
\hline & & & \\
\hline & & & \\
\hline & & & \\
\hline & & & \\
\hline & & & \\
\hline
\end{tabular}




\begin{tabular}{|c|c|c|c|}
\hline 6 & -2.165088 & 0.672914 & 0.000038 \\
\hline 6 & -2.124995 & -0.72338 & 0.000022 \\
\hline 6 & -0.907999 & -1.356958 & 0.000078 \\
\hline 7 & 0.214073 & -0.620675 & 0.000036 \\
\hline 1 & -3.120393 & 1.179313 & 0.000138 \\
\hline 1 & -1.005795 & 2.491245 & -0.000216 \\
\hline 1 & -3.029736 & -1.31125 & -0.000059 \\
\hline 1 & -0.786953 & -2.430819 & -0.000095 \\
\hline 6 & 1.591842 & 1.192371 & 0.000011 \\
\hline 6 & \begin{tabular}{|l|}
2.394142 \\
\end{tabular} & 0.126489 & 0.000158 \\
\hline 6 & 1.593138 & -1.1324 & -0.000096 \\
\hline 1 & 1.876025 & 2.231854 & -0.000103 \\
\hline 1 & 3.4727 & 0.125133 & 0.000363 \\
\hline 1 & \begin{tabular}{|l|}
1.758424 \\
\end{tabular} & -1.745123 & -0.887893 \\
\hline \multirow[t]{5}{*}{1} & 1.758416 & -1.74604 & 0.886975 \\
\hline & -364.14676 & & \\
\hline & \begin{tabular}{|l|l|}
0.143397 \\
\end{tabular} & & \\
\hline & \begin{tabular}{|l|}
0.150627 \\
\end{tabular} & & \\
\hline & 0 & & \\
\hline CO (cinfv) & $X$ & $\mathrm{Y}$ & $\mathrm{Z}$ \\
\hline Atomic \# & 0 & 0 & -0.648343 \\
\hline 6 & 0 & 0 & 0.473012 \\
\hline \multicolumn{4}{|l|}{8} \\
\hline & -113.320241 & & \\
\hline & \begin{tabular}{|l}
0.005177 \\
\end{tabular} & & \\
\hline & 0.008482 & & \\
\hline & 0 & & \\
\hline
\end{tabular}




\begin{tabular}{lccc}
\multicolumn{5}{c}{ 1,2-didehydropyridinium cation } \\
UB3LYP/cc-pVTZ \\
\multicolumn{4}{c}{ 1A' singlet state (Cs) } \\
7 & -1.116453 & 0.806467 & 0.000000 \\
6 & 0.000000 & 1.219616 & 0.000000 \\
6 & 1.279808 & 0.795121 & 0.000000 \\
6 & 1.132032 & -0.616106 & 0.000000 \\
6 & -0.110447 & -1.266402 & 0.000000 \\
6 & -1.299599 & -0.548573 & 0.000000 \\
1 & 2.207275 & 1.341896 & 0.000000 \\
1 & 2.050010 & -1.193498 & 0.000000 \\
1 & -0.152192 & -2.346992 & 0.000000 \\
1 & -2.300688 & -0.948606 & 0.000000
\end{tabular}

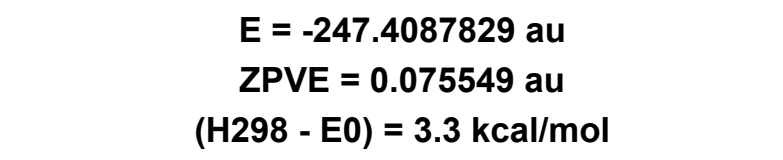

\section{1,2-didehydropyridinium cation} UB3LYP/cc-pVTZ

3A' triplet state (Cs)

$\begin{array}{llll}6 & -1.267165 & -0.700288 & 0.000000 \\ 6 & -1.241049 & 0.665432 & 0.000000 \\ 6 & 0.000000 & 1.311849 & 0.000000 \\ 6 & 1.181250 & 0.580237 & 0.000000 \\ 6 & 1.102418 & -0.818914 & 0.000000 \\ 7 & -0.090485 & -1.342105 & 0.000000 \\ 1 & 0.038541 & 2.393476 & 0.000000 \\ 1 & -2.182477 & 1.209460 & 0.000000 \\ 1 & 2.157685 & 1.042813 & 0.000000 \\ 1 & 1.966923 & -1.480913 & 0.000000\end{array}$

\begin{tabular}{|c|c|c|c|}
\hline & \multicolumn{3}{|c|}{$\begin{array}{c}\text { ZPVE }=0.079105 \mathrm{au} \\
(\mathrm{H} 298-\mathrm{E} 0)=3.3 \mathrm{kcal} / \mathrm{mol}\end{array}$} \\
\hline & $\begin{array}{r}2,3-d i d e \\
1 A\end{array}$ & $\begin{array}{l}\text { opyridiniur } \\
\text { YP/cc-pVT } \\
\text { glet state (C }\end{array}$ & tion \\
\hline 1 & -2.075986 & 1.158901 & 0.000000 \\
\hline 7 & -1.218428 & 0.614110 & 0.000000 \\
\hline 6 & -1.182503 & -0.713037 & 0.000000 \\
\hline 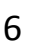 & -0.110243 & -1.336095 & 0.000000 \\
\hline
\end{tabular}




$\begin{array}{rrrr}6 & 1.184935 & -0.913331 & 0.000000 \\ 6 & 1.176347 & 0.506424 & 0.000000 \\ 6 & 0.000000 & 1.236927 & 0.000000 \\ 1 & 2.092026 & -1.500017 & 0.000000 \\ 1 & 2.117831 & 1.039632 & 0.000000 \\ 1 & -0.016085 & 2.317382 & 0.000000\end{array}$

$E=-247.3719373 \mathrm{au}$

ZPVE $=0.077067$ au

$(\mathrm{H} 298-\mathrm{EO})=3.3 \mathrm{kcal} / \mathrm{mol}$

\section{2,3-didehydropyridinium cation}

UB3LYP/cc-pVTZ

$3 A^{\prime}$ triplet state (Cs)

$\begin{array}{llll}1 & -2.055693 & 1.114140 & 0.000000\end{array}$

$\begin{array}{llll}7 & -1.180501 & 0.593780 & 0.000000\end{array}$

$\begin{array}{llll}6 & -1.227975 & -0.732676 & 0.000000\end{array}$

$\begin{array}{llll}6 & 0.000000 & 1.271304 & 0.000000\end{array}$

$\begin{array}{llll}6 & -0.066771 & -1.475952 & 0.000000\end{array}$

$\begin{array}{llll}6 & 1.178218 & 0.562577 & 0.000000\end{array}$

$\begin{array}{llll}6 & 1.148508 & -0.841680 & 0.000000\end{array}$

$\begin{array}{llll}1 & -0.061126 & 2.349104 & 0.000000\end{array}$

$\begin{array}{llll}1 & 2.118543 & 1.094278 & 0.000000\end{array}$

$\begin{array}{llll}1 & 2.069907 & -1.415414 & 0.000000\end{array}$

$E=-247.3339943 \mathrm{au}$

ZPVE $=0.076266$ au

$(\mathrm{H} 298-\mathrm{EO})=3.3 \mathrm{kcal} / \mathrm{mol}$

\section{3,4-didehydropyridinium cation}

UB3LYP/cc-pVTZ

1A' singlet state (Cs)

$\begin{array}{llll}1 & 0.081467 & 2.191068 & 0.000000 \\ 7 & 0.000000 & 1.178811 & 0.000000 \\ 6 & -1.260218 & 0.643200 & 0.000000 \\ 6 & -1.233354 & -0.720089 & 0.000000 \\ 6 & -0.157184 & -1.339663 & 0.000000 \\ 6 & 1.148104 & -0.931513 & 0.000000 \\ 6 & 1.151902 & 0.466188 & 0.000000 \\ 1 & -2.096844 & 1.326263 & 0.000000 \\ 1 & 2.052697 & -1.519315 & 0.000000 \\ 1 & 2.067181 & 1.041573 & 0.000000\end{array}$




$$
\begin{gathered}
E=-247.3874145 \mathrm{au} \\
\text { ZPVE }=0.077525 \mathrm{au} \\
(\mathrm{H} 298-\mathrm{E} 0)=3.3 \mathrm{kcal} / \mathrm{mol}
\end{gathered}
$$

\section{3,4-didehydropyridinium cation}

UB3LYP/cc-pVTZ

3A' triplet state (Cs)

$\begin{array}{lccc}1 & 0.051047 & 2.225023 & 0.000000 \\ 7 & 0.000000 & 1.211448 & 0.000000 \\ 6 & -1.224496 & 0.629902 & 0.000000 \\ 6 & 1.152399 & 0.515084 & 0.000000 \\ 6 & -1.283807 & -0.732581 & 0.000000 \\ 6 & 1.121261 & -0.870617 & 0.000000 \\ 6 & -0.110344 & -1.480082 & 0.000000 \\ 1 & -2.092212 & 1.276200 & 0.000000 \\ 1 & 2.068076 & 1.088131 & 0.000000 \\ 1 & 2.043014 & -1.439722 & 0.000000\end{array}$

$$
\begin{gathered}
E=-247.3365123 \mathrm{au} \\
\text { ZPVE }=0.076567 \mathrm{au} \\
(\mathrm{H} 298-\mathrm{E} 0)=3.3 \mathrm{kcal} / \mathrm{mol}
\end{gathered}
$$

\section{References:}

(1) Mariet, N.; Ibrahim-Ouali, M.; Parrain, J.-L.; Santelli, M. Structure of Pyridynes and Pyridyne NOxides and Their Cycloaddition to Ketene Dialkyl Acetals. J. Mol. Struct. Theochem. 2004, 679 (12), 53-58.

(2) Hoffmann, R. W. Dehydrobenzene and Cycloalkynes; Verlag Chemie: Academic Press: Weinheim/Bergstr.; New York; London, 1967.

(3) Goetz, A. E.; Bronner, S. M.; Cisneros, J. D.; Melamed, J. M.; Paton, R. S.; Houk, K. N.; Garg, N. K. An Efficient Computational Model to Predict the Synthetic Utility of Heterocyclic Arynes. Angew. Chem. Int. Ed. 2012, 51 (11), 2758-2762.

(4) Wolthuis, E.; Bouma, B.; Modderman, J.; Sytsma, L. Cleavage of Tetrahydrofuran by Benzyne. Tetrahedron Lett. 1970, 11 (6), 407-408.

(5) Brewer, J. P. N.; Heaney, H.; Jablonski, J. M. Aryne Chemistry Part XV. Tetrahedron Lett. 1968, 9 (42), 4455-4456.

(6) Duboudin, J.-G.; Jousseaume, B.; Pinet, M. Evidence for [2 + 2] and [4 + 2] Cycloadditions of Allylic Grignard Reagents to Benzyne. J. Chem. Soc., Chem. Commun. 1977, No. 13, 454.

(7) Tabushi, I.; Okazaki, K.; Oda, R. Reaction of Benzyne with Disulfides. Tetrahedron Lett. 1967, 8 (37), 3591-3593. 